\title{
Cathepsin K-deficient osteocytes prevent lactation-induced bone loss and parathyroid hormone suppression
}

\author{
Sutada Lotinun, ${ }^{1,2}$ Yoshihito Ishihara, ${ }^{1}$ Kenichi Nagano, ${ }^{1}$ Riku Kiviranta, ${ }^{1,3}$ Vincent T. Carpentier, ${ }^{1}$ Lynn Neff, ${ }^{1}$ Virginia Parkman, ${ }^{1}$ \\ Noriko Ide, ${ }^{1}$ Dorothy Hu, ${ }^{1}$ Pamela Dann, ${ }^{4}$ Daniel Brooks, ${ }^{5}$ Mary L. Bouxsein, ${ }^{5}$ John Wysolmerski, ${ }^{4}$ Francesca Gori, ${ }^{1}$ and Roland Baron ${ }^{1,6}$
}

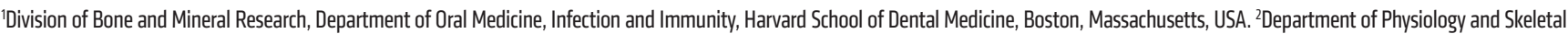
Disorders Research Unit, Faculty of Dentistry, Chulalongkorn University, Bangkok, Thailand. ${ }^{3}$ Departments of Medical Biochemistry and Genetics and Medicine, University of Turku, Turku, Finland. ${ }^{4}$ Section of Endocrinology and Metabolism, Department of Internal Medicine, Yale University School of Medicine, New Haven, Connecticut, USA. ${ }^{5}$ Center for Advanced Orthopedic Studies, Beth Israel Deaconess Medical Center and Harvard Medical School, Boston, Massachusetts, USA. ${ }^{6}$ Harvard Medical School, Department of Medicine, Endocrine Unit, Massachusetts Ceneral Hospital, Boston, Massachusetts, USA.

\begin{abstract}
Lactation induces bone loss to provide sufficient calcium in the milk, a process that involves osteoclastic bone resorption but also osteocytes and perilacunar resorption. The exact mechanisms by which osteocytes contribute to bone loss remain elusive. Osteocytes express genes required in osteoclasts for bone resorption, including cathepsin K (Ctsk), and lactation elevates their expression. We show that Ctsk deletion in osteocytes prevented the increase in osteocyte lacunar area seen during lactation, as well as the effects of lactation to increase osteoclast numbers and decrease trabecular bone volume, cortical thickness, and mechanical properties. In addition, we show that Ctsk deletion in osteocytes increased bone parathyroid hormone-related peptide (PTHrP) and prevented the decrease in serum parathyroid hormone (PTH) induced by lactation, but amplified the increase in serum 1,25-dyhydroxyvitamin $\mathrm{D}\left[1,25(\mathrm{OH})_{2} \mathrm{D}\right]$. The net result of these changes is to maintain serum and milk calcium levels in the normal range, ensuring normal offspring skeletal development. Our studies confirm the fundamental role of osteocytic perilacunar remodeling in physiological states of lactation and provide genetic evidence that osteocyte-derived Ctsk contributes not only to osteocyte perilacunar remodeling, but also to the regulation of PTH, PTHrP, 1,25(OH) 2 D, osteoclastogenesis, and bone loss in response to the high calcium demand associated with lactation.
\end{abstract}

\section{Introduction}

Cathepsin $\mathrm{K}$ (Ctsk) is highly expressed in osteoclasts and is secreted in the bone-resorbing compartment, where it degrades the organic bone matrix after dissolution of the mineral phase at low $\mathrm{pH}$ during bone resorption $(1,2)$. Consequently, global deletion, osteoclast-targeted deletion, or pharmacological inhibition of Ctsk impairs bone resorption while increasing bone formation and results in a high bone mass (2-6). In all these situations, this is associated with high RANKL levels, increasing the number of otherwise functionally impaired osteoclasts (4-6) and mimicking the osteoclast-rich osteopetrotic phenotype observed in pycnodysostosis (7-9). Although osteocytes have been shown to resorb their perilacunar space (10-17), whether Ctsk is involved in this process is not known. Interestingly, during lactation in mice, a subpopulation of osteocytes expresses high mRNA and protein levels of Ctsk and other genes expressed in osteoclasts and linked to bone resorption

\section{Related Commentary: p. 3041}

Authorship note: FG and RB are co-senior authors.

Conflict of interest: RB and MB were members of the Merck Osteoporosis Global Advisory Board and received honoraria and research support from this company. Copyright: (๖) 2019, American Society for Clinical Investigation.

Submitted: June 15, 2018; Accepted: May 10, 2019; Published: June 24, 2019

Reference information: / Clin Invest. 2019;129(8):3058-3071.

https://doi.org/10.1172/JCl122936. such as tartrate-resistant acid phosphatase (Trap), carbonic anhydrase, or the proton pump V-ATPase $(18,19)$. This suggests that, at least under high systemic calcium demand, osteocytes are able to turn on a set of genes required for bone resorption to enlarge their lacunae. More recently, it was shown that these cells can also acidify their lacunar space (19), thus suggesting an osteoclast-like resorptive phenotype and supporting the potential relevance of osteocytes in calcium mobilization during lactation (15).

It is known that in order to provide adequate amounts of calcium to rapidly growing pups, lactation induces an increase in osteoclast numbers and bone resorption, resulting in a substantial mobilization of calcium into the milk at the expense of the skeleton $(15,20)$. Accordingly, lactating mice lose up to $30 \%$ of their bone mass over a 2-week period of breastfeeding (21-23). This rapid decrease in bone mass is, however, reversible, and bone mass is rapidly restored after weaning in many species (20, 23-26). Although this mobilization of calcium and the resulting bone loss have for the most part been attributed to low estrogen levels and an increase in parathyroid hormone-related peptide (PTHrP) production by the mammary glands, both resulting in an increase in osteoclast numbers $(23,24)$, the exact mechanisms underlying bone loss during lactation are incompletely understood. In particular, whether osteocyte perilacunar resorption plays a role in calcium mobilization and in the induction of osteoclastogenesis is not known. Thus, like osteoclastic bone resorption, Ctsk may be involved in bone matrix degradation during the process of osteo- 
cyte perilacunar resorption and, therefore, in the mobilization of calcium for the milk during lactation. In addition to its potential role in calcium mobilization, lactation-induced osteocytic perilacunar resorption negatively affects bone biomechanical properties by increasing the volume of the osteocytic network (27).

Although several studies have examined osteocyte responses to hormones, cytokines, mechanical loading, and lactation, as well as the influence of osteocytes on bone remodeling and the bone marrow (BM) microenvironment $(18,28-34)$, the feedback influence of perilacunar resorption on osteocytes themselves and the possibility that osteocyte-mediated changes in the periosteocytic matrix contribute to the way in which they regulate osteoclast differentiation and bone remodeling had not been investigated. Recently, a study by Dole et al. (17) reported that TGF- $\beta$ receptor 2 (TGF- $\beta$ R2) in osteocytes is required to maintain perilacunar remodeling, bone mass, and bone quality, but whether perilacunar remodeling affects osteocyte regulatory behavior was not explored.

Here, we hypothesized that while performing perilacunar resorption during lactation, osteocytes may be affected by the changes occurring in their lacunar microenvironment as a consequence of perilacunar resorption itself. This may in turn affect the way in which osteocytes regulate osteoclast differentiation and thereby calcium mobilization and bone loss during lactation. According to this hypothesis, deletion of Ctsk in osteocytes could not only prevent matrix degradation around osteocytes and perilacunar resorption but also alter the recruitment of osteoclasts, bone loss, and calcium metabolism responses during lactation.

To test this hypothesis, we targeted the deletion of Ctsk in osteocytes by crossing 9.6-kb Dmp1Cre-transgenic mice with $C t s k^{f / f l}$ mice (Ctsk ${ }^{o c y}$ mice). Deletion of Ctsk in osteocytes prevented the increase in the osteocyte lacunar area, osteoclast numbers, and bone resorption, preventing the bone loss seen in lactating control mice at both cortical and cancellous sites. In addition, we found that $C t s k$ deletion in osteocytes protected against the alteration of some biomechanical properties of the femur. Interestingly, higher levels of serum parathyroid hormone (PTH), bone PTHrP, and serum 1,25 -dyhydroxyvitamin $\mathrm{D}\left[1,25(\mathrm{OH})_{2} \mathrm{D}\right]$ in Ctsk $^{o c y}$ lactating mice compared with levels in control lactating mice allowed the levels of serum C-terminal telopeptides of type I collagen (CTX1) and calcium in serum and milk to remain normal, ensuring normal skeletal development in the offsprings of mothers lacking Ctsk in osteocytes. These results suggest that, in mice, lacunar Ctsk and/ or the changes it induces during lactation-induced perilacunar resorption generate signals that modify osteocytic regulation of osteoclast differentiation and mineral metabolism to ensure the appropriate mobilization of the calcium required for milk production and skeletal development in offspring.

\section{Results}

At steady state, Ctsk ${ }^{o c y}$ mice exhibit normal skeletal homeostasis and remodeling. As previously reported (18), a number of osteocytes expressed Ctsk at steady state in control mice (Figure 1A). To determine whether Ctsk in osteocytes affects bone homeostasis at steady state, we crossed $C t s k^{f / f l}$ mice with transgenic mice expressing Cre recombinase under the control of 9.6-kb Dmp1 regulatory elements. Dmp1Cre Ctsk $k^{f l f l}\left(C t s k^{o c y}\right)$ mice were born at the expected
Mendelian ratio and had no obvious skeletal phenotype at birth, as assessed by gross examination, compared with control littermates $\left(C t s k^{f / f l}\right)$ (data not shown). Ctsk ${ }^{o c y}$ mice showed efficient $C t s k$ exon 5 genomic excision and a significant Ctsk deletion in long bones and muscle, but not in other tissues (Supplemental Figure 1A; supplemental material available online with this article; https://doi. org/10.1172/JCI122936DS1), as previously reported (35), and a significant reduction of Ctsk protein levels in osteocytes and Ctsk mRNA levels in marrow-depleted long bone midshafts (Figure 1, $\mathrm{A}$ and $\mathrm{B})$. Immunohistochemistry and quantitative reverse transcription PCR (qRT-PCR) confirmed the specificity of Dmp1Cre to osteocytes, with osteoclast Ctsk remaining unaffected (Figure 1, C and D). Furthermore, in our hands, there was no Cre recombination (Supplemental Figure 1B) or Ctsk deletion in the mammary glands of $C t s k^{o c y}$ mice, whether or not the mice were lactating (virgin $C t s k^{f l / f l}=1.0 \pm 0.1$, virgin $C t s k^{o c y}=0.72 \pm 0.1$, lactating $C t s k^{f l / f l}=$ $0.6 \pm 0.2$, and lactating $C t s k^{o c y}=1.1 \pm 0.4 ; n=4-5$, mean \pm SEM). MicroCT $(\mu \mathrm{CT})$ and histomorphometric analyses indicated that deletion of Ctsk in osteocytes did not alter cortical or cancellous bone in 12-week-old female mice under steady-state conditions (Figure 1E and Supplemental Tables 1 and 2). As seen in the female mice, except for an increase in trabecular thickness, we observed no changes in other parameters in the Ctsk ${ }^{o c y}$ males compared with control littermates (Supplemental Table 3 and data not shown). Interestingly, however, and as detailed below, Ctsk deletion in osteocytes altered the level of expression of several genes relevant to bone homeostasis in osteocytes themselves.

Osteocyte-specific deletion of Ctskpreventslactation-induced osteocytic perilacunar resorption. We then determined whether deletion of Ctsk in osteocytes would affect the skeletal response during lactation in female mice. Deletion of Ctsk in osteocytes had no effect on the femoral diaphysis osteocyte lacunar area in virgin mice as assessed by BSEM, nor on osteocyte numbers or density (Figure 2A and Supplemental Table 4). In contrast, and as previously shown $(15,18-20)$, osteocyte perilacunar resorption was increased during lactation in control mice, resulting in a significant increase in the osteocyte lacunar area and osteocyte lacunar perimeter (Figure 2B and Supplemental Table 4). Importantly, these changes were prevented by deletion of Ctsk in osteocytes (Figure 2B and Supplemental Table 4). In control mice, the number of osteocytes per unit bone volume (osteocyte density) was significantly increased (Supplemental Table 4) during the experimental period (which always covers 19 days of pregnancy and 12 days of lactation) $(18,19,36)$, suggesting a decrease in the amount of matrix produced by each cell during the process of bone formation that led to embedment of the osteocytes. This increase in osteocyte density was partially prevented by Ctsk deletion (Supplemental Table 4). Histologically, no striking changes in the canalicular network were observed at steady state, during lactation, or between $C t s k^{o c y}$ and $C t s k^{f / f l}$ females, as indicated by silver nitrate staining (Figure 2C). These findings therefore provide direct evidence that during lactation, a time of high calcium demand, Ctsk is required in osteocytes to enable the process of perilacunar resorption.

Osteocyte-specific deletion of Ctsk prevents bone loss in lactating mice. To examine whether Ctsk in osteocytes also contributes to bone loss during lactation, we measured bone turnover and homeostasis in virgin and lactating $C t s k^{o c y}$ mice and control litter- 


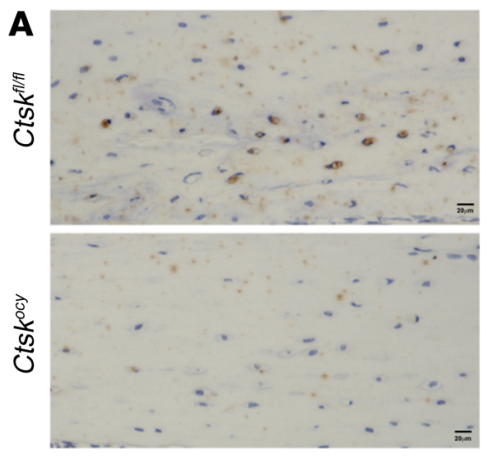

B
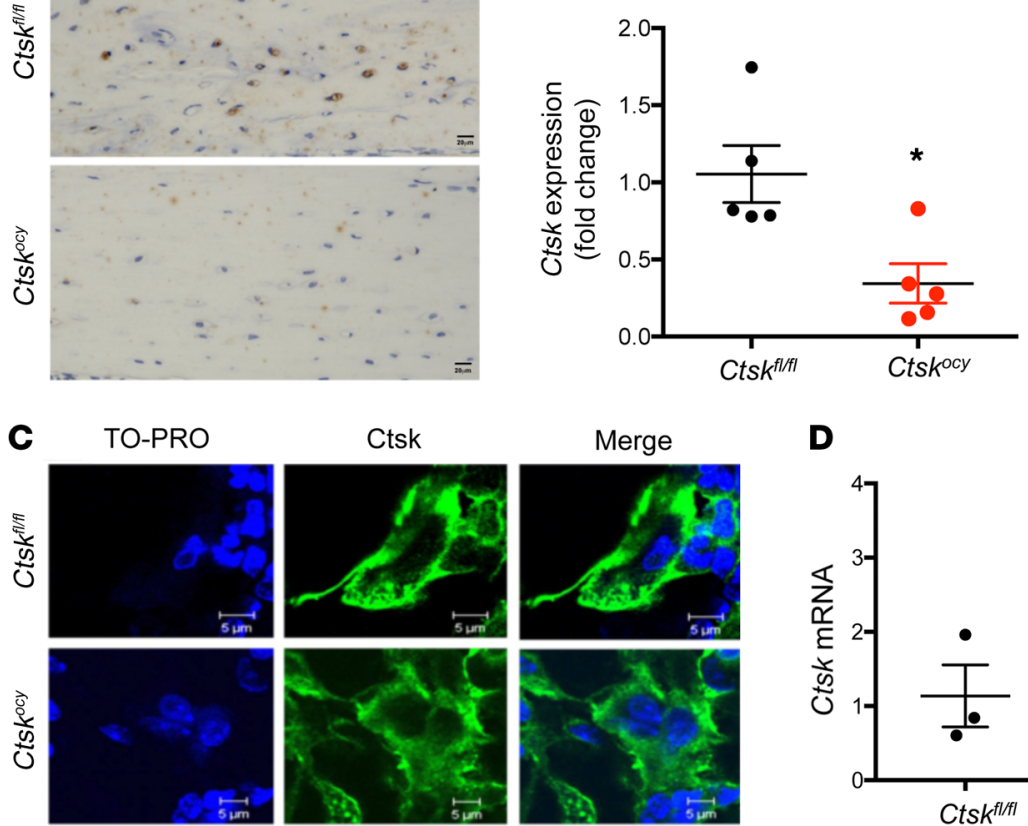

D
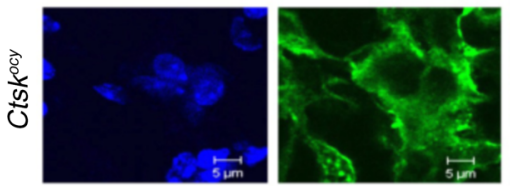

$\mathbf{E}$
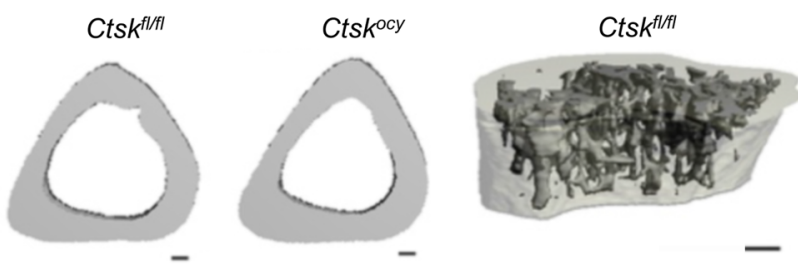

Figure 1. Normal skeletal homeostasis in mice with osteocyte-specific deletion of Ctsk at steady state. (A) Representative images of Ctsk immunostaining in osteocytes from Ctsk $k^{f l / f l}$ and $C t s k^{o c y}$ mice $(n=3$ per group). Scale bars: $20 \mu \mathrm{m}$. (B) qRT-PCR analysis of Ctsk expression in BM-depleted distal femur from 12-week-old Ctsk flffl (black $^{\text {fla }}$ dots) and $C t s k^{o c y}$ (red dots) mice. ( $n=5$ per group). ${ }^{*} P<0.05$, by Student's $t$ test versus $C t s k^{f l / f l}$ mice. Data are expressed as the mean \pm SEM. (C) Representative images of Ctsk immunostaining in osteoclasts from Ctsk ${ }^{f / f f l}$ and $C t s k^{o c y}$ mice $(n=3)$. Scale bars: $5 \mu \mathrm{m}$. (D) qRT-PCR analysis of relative Ctsk expression in osteoclasts. Ctsk ${ }^{f l / f l}$ (black dots) and Ctsk ${ }^{o c y}$ (red dots) mice ( $n=3$ per group). Data are expressed as the mean \pm SEM. (E) Representative $\mu \mathrm{CT}$ images of the cortical area and the trabecular microarchitecture of tibiae from 12-week-old female Ctsk $k^{f l / f l}$ and Ctsk ${ }^{\text {ocy }}$ mice ( $n=6$ per group). Scale bars: $100 \mu \mathrm{m}$. mates. As shown in Supplemental Table 1 and Figure 3, $\mu \mathrm{CT}$ analysis revealed that when Ctsk was present in osteocytes, a 12-day lactation period significantly decreased cortical bone volume and cortical thickness in control females. Cancellous bone volume was also significantly (Supplemental Table 1 and Figure 3) lower in the lactating control mice than in the virgin mice (Supplemental Table 1). Strikingly, these changes were not observed in Ctsk ${ }^{\text {ocy }}$ mice, indicating that the absence of Ctsk in osteocytes prevented the effects of lactation on the skeleton. We also measured vertebral bone mineral content by dual-energy x-ray absorptiometry (DEXA) and found a similar protection from bone loss in lactating $\mathrm{Ctsk}^{o c y}$ mice compared with virgin mice $(-44.5 \% \pm 3.2 \%$ in control lactating mice vs. $-6.4 \% \pm 5.5 \%$ in Ctsk $^{o c y}$ lactating mice, $P<$ $0.0001, n=5)$. Thus, Ctsk expressed by osteocytes contributes to the negative effects of lactation on the skeleton.

To determine whether deletion of Ctsk in osteocytes affects bone surface remodeling, we performed dynamic histomorphometry. As expected, lactation significantly decreased trabecular bone volume and trabecular thickness, confirming the $\mu \mathrm{CT}$ results (Supplemental Table 2 and Figure 4). This loss of bone was due to a significant increase in osteoclast numbers, with no significant changes in the bone formation rate in control lactating mice (Supplemental Table 2 and Figure 4). In contrast, deletion of Ctsk in osteocytes prevented the decrease in bone volume, confirming the $\mu \mathrm{CT}$ results, and trabecular thickness. Most strikingly, Ctsk deletion in osteocytes also prevented the lactation-dependent increase in osteoclast numbers and maintained bone formation parameters that were modestly decreased in lactating control mice, within the normal range, except for a significant increase in mineralizing surface and osteoid thickness (Figure 4 and Supplemental Table 2). These findings along trabecular bone surfaces were confirmed along endocortical surfaces in a separate cohort (data not shown). These results demonstrated that $C t s k^{o c y}$ mice are protected from lactation-induced increases in osteoclastogenesis. Although we observed no significant changes in procollagen type $1 \mathrm{~N}$-terminal propeptide (P1NP) between genotypes during lactation, serum CTX1 levels were significantly increased in control lactating mice but remained at basal levels in Ctsk ${ }^{o c y}$ lactating mice, consistent with the lower perilacunar resorption and osteoclast numbers observed in $C t s k^{o c y}$ lactating mice (Figure 5 and Supplemental Table 5).

Osteocyte-expressed Ctsk regulates the expression of osteocytic genes involved in bone formation and resorption. The deletion of Ctsk was targeted to osteocytes, which are major regulators of bone remodeling $(16,37)$. Since we observed significant changes in remodeling activity, we then determined whether deletion of Ctsk affects the expression of key regulatory genes in osteocytes. In agreement with previous observations in global or osteoclast-targeted Ctsk deletion (4-6), virgin Ctsk ${ }^{o c y}$ mice had higher Rankl levels than did virgin $C t s k^{f l / f l}$ mice. However, Opg mRNA expression was also higher (2.6-fold) in virgin Ctsk ${ }^{o c y}$ mice compared with expression in virgin $C t s k^{f / f l}$ mice, explaining why the number of osteoclasts was not increased in Ctsk ${ }^{o c y}$ mice. As expected, lacta- 

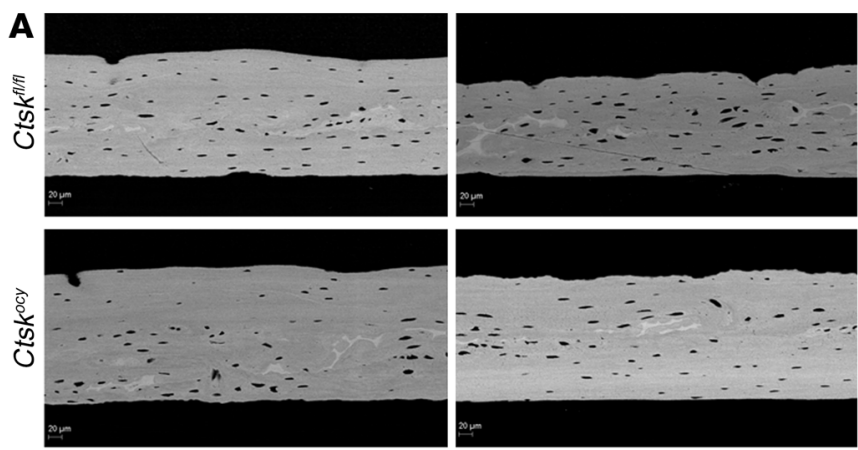

B Osteocyte lacunar area $\left(\mu \mathrm{m}^{2}\right)$

Osteocyte lacunar perimeter $(\mu \mathrm{m})$
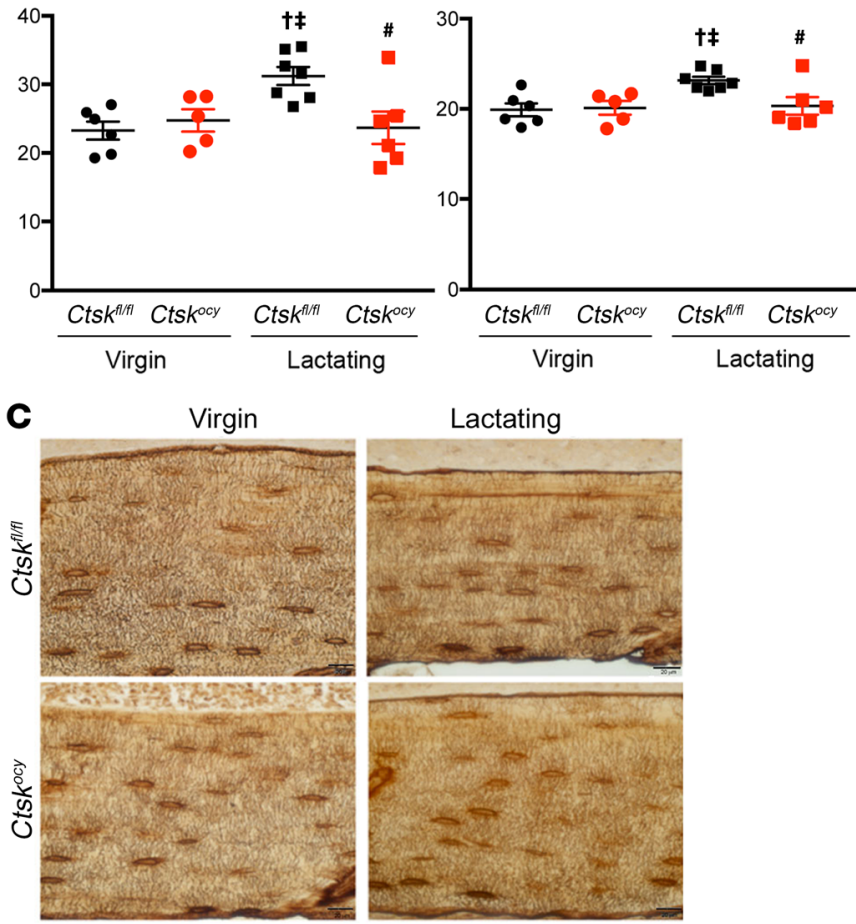

tion led to a marked increase in Rankl mRNA expression in Ctsk ${ }^{f / f l}$ mice, whereas in $C t s k^{o c y}$ mice its expression remained high but was significantly lower than in lactating control mice and at levels similar to those detected in virgin Ctsk ${ }^{o c y}$ mice (Figure 6A and Supplemental Table 6). Furthermore, while Opg mRNA expression was not changed during lactation in $\mathrm{Ctsk}^{\mathrm{fl} / \mathrm{fl}}$ mice, it decreased by approximately 2-fold in lactating Ctsk ${ }^{o c y}$ mice (Figure 6A and Supplemental Table 6). Consequently, the Rankl/Opg ratio, which was not significantly increased in virgin $C t s k^{o c y}$ mice, increased by less than 2 -fold during lactation but increased by 8 -fold in lactating control mice, reaching a significantly higher level than that seen in $C t s k^{o c y}$ mice (Figure 6A and Supplemental Table 6). Since we were targeting osteocytes in this study, we also measured sclerostin (Sost), fibroblast growth factor 23 (Fgf23), and PhtrP mRNA levels in these BM-flushed cortical bone samples. Deletion of Ctsk in osteocytes significantly (Supplemental Table 6) decreased Sost mRNA levels in both virgin and lactating $C t s k^{o c y}$ mice compared with levels in $C t s k^{f / f l}$ mice, whereas it was profoundly repressed during lactation in both $C t s k^{f / f l}$ and Ctsk ${ }^{o c y}$ mice (Figure 6B and Supplemental Table 6), probably as a consequence of the changes
Figure 2. Deletion of Ctsk in osteocytes decreases perilacunar remodeling during lactation. (A) Representative BSEM images (original magnification, $\times 500)$ of the femur diaphysis of virgin and lactating Ctsk $k^{f / f l}$ and $C t s k^{\text {ocy }}$ mice ( $n=5-7$ per group). (B) Measurements of the single osteocyte lacunar area and perimeter in virgin and lactating $C t s k^{f l / f l}$ (black dots and squares, respectively) and Ctsk ${ }^{\text {ocy }}$ (red dots and squares, respectively) mice. Data are expressed as the mean \pm SEM ( $n=5-7$ per group). ${ }^{\dagger} P<0.05$ versus virgin $C t s k^{f l f f l}$ mice; ${ }^{\ddagger} P<0.05$ versus virgin Cts $k^{\text {ocy }}$ mice; and ${ }^{\#} P<0.05$ versus lactating $C$ tsk $^{f / / f l}$ mice; 2-way ANOVA followed by Fisher's PLSD. (C) Representative images of silver nitrate staining showing osteocyte canalicular networking in cortical bone from virgin and lactating Cts $k^{f / f l}$ and $C t s k^{o c y}$ mice ( $n=3$ per group). Scale bars: $20 \mu \mathrm{m}$.

in PTH and PTHrP levels (38). Indeed, bone PthrP mRNA levels were 6-fold higher in virgin $C t s k^{o c y}$ mice than in virgin $C t s k^{f l / f l}$ mice, and although these levels increased by 11-fold during lactation in $C t s k^{f l / f l}$ mice, they simply remained high in Ctsk ${ }^{o c y}$ mice (Figure 6A and Supplemental Table 6). Given the known role of matrix metallopeptidase 13 (Mmp13) in bone extracellular matrix and osteocytic perilacunar remodeling (36), we also assessed whether deletion of Ctsk in osteocytes affects the expression of this metalloproteinase. As shown in Figure 6A and Supplemental Table 6, Mmp13 levels were similar in control and Ctsk ${ }^{\text {ocy }}$ bone and increased by 3- and 6-fold, respectively, during lactation. Histological evaluation of sclerostin confirmed the decrease seen in Sost mRNA levels in virgin $C t s k^{o c y}$ and lactating Ctsk $k^{f l f l}$ and $C t s k^{o c y}$ mice (Figure 6B). Interestingly, osteocyte Fgf 23 mRNA levels were significantly decreased by Ctsk deletion and by lactation in control mice, but we observed no difference in Ctsk ${ }^{o c y}$ lactating mice (Figure 6A and Supplemental Table 6). Importantly, qRT-PCR evaluation of Ctsk mRNA and protein expression levels as well as TRAP activity confirmed a lactation-dependent increase in Ctsk levels and the percentage of $\mathrm{TRAP}^{+}$osteocytes in lactating $\mathrm{Ctsk}^{f l / f l}$ mice, whereas the number of $\mathrm{TRAP}^{+}$osteocytes was high in $C t s k^{o c y}$ mice, independent of lactation (Figure 6, A and C-E).

To determine which changes were cell autonomous, we next determined whether Ctsk regulates gene expression in osteocytes in vitro. For this purpose, and because Ctsk is involved in collagen degradation, we used the Ocy454 cell line cultured on collagen (39). Ocy 454 cells express Ctsk, whose expression increases markedly during cell differentiation (Figure 7). Ctsk shRNA in Ocy454 cells significantly reduced the expression of Ctsk, as indicated by qRT-PCR, Western blotting, and immunohistochemical analyses (Figure 7, A-C). Similar findings were observed with 2 separate shRNAs targeting distinct regions of Ctsk (Supplemental Figure 2, A and B). The effect of Ctsk silencing on the expression of Rankl, osteoprotegerin $(\mathrm{Opg})$, and Sost confirmed our in vivo cortical bone mRNA observations (Figure 7D), except after 14 days in culture, when Sost mRNA was unchanged by Ctsk knockdown. Together with the in vivo data, these results suggest that osteocyte-expressed Ctsk acts in a cell-autonomous autocrine manner to regulate osteocytic genes involved in the regulation of bone remodeling.

Deletion of Ctsk in osteocytes partially protects lactating mothers against lactation-induced bone fragility. Finally, and because lactation has been shown to significantly increase bone fragility in mice (27), we sought to determine whether the deleterious effects of lactation on bone mechanical properties were prevented in $C t s k^{o c y}$ lactating mice. For this purpose, we tested the mechanical prop- 
A

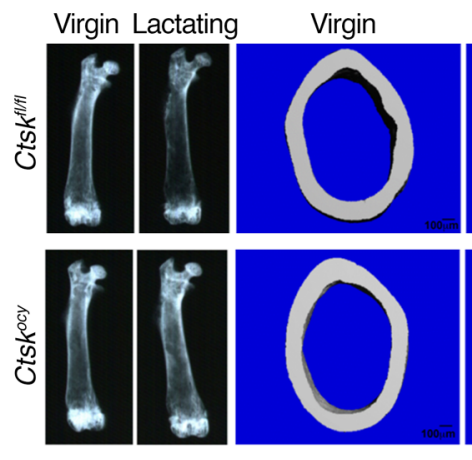

B

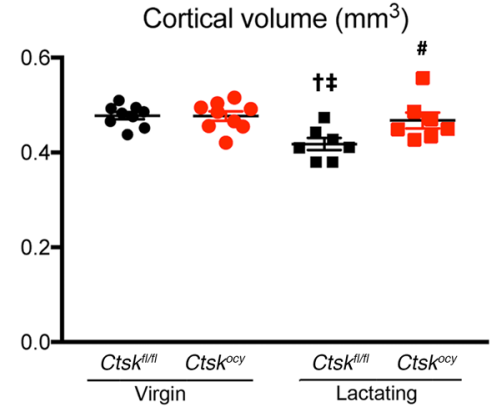

C
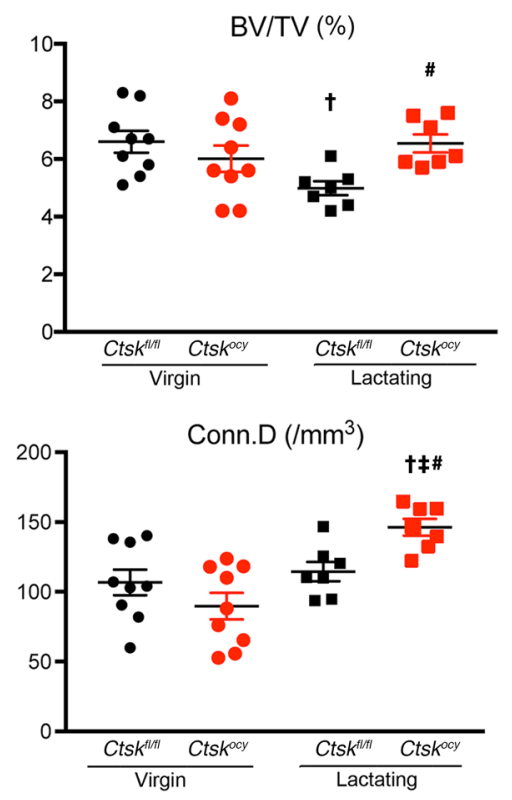

Lactating

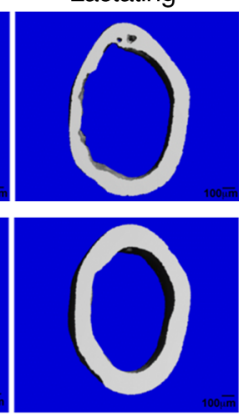

Virgin
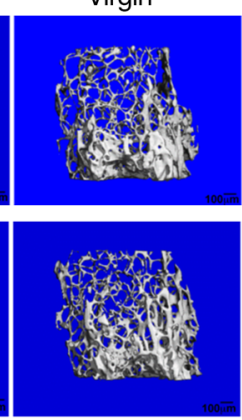

Cortical Thickness (mm)

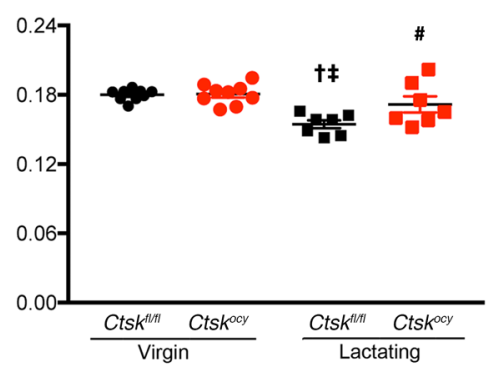

Tb.Th (mm)
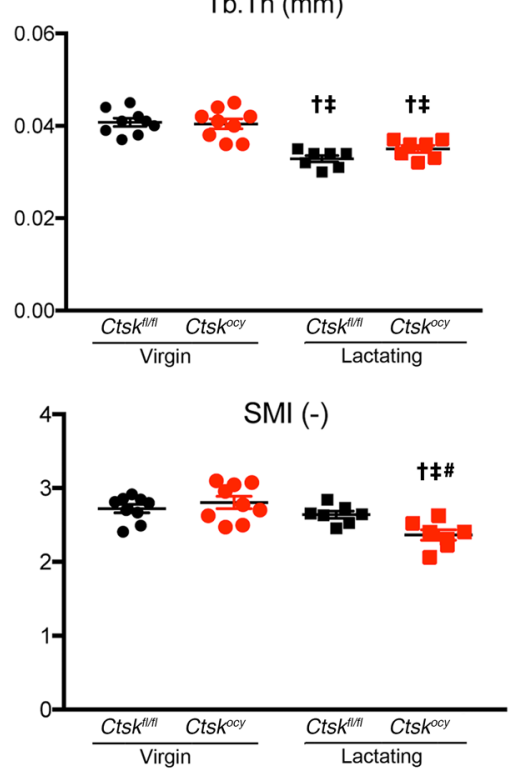

Lactating
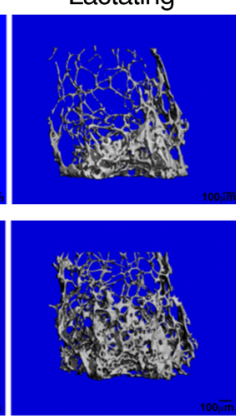

Figure 3. Deletion of Ctsk in osteocytes preserves cortical and cancellous bone during lactation. (A) Representative $x$-ray and $\mu \mathrm{CT}$ images of virgin and lactating $C t s k^{f l / f l}$ and Ctsk $k^{\text {ocy }}$ femurs ( $n=7-9$ per group). Scale bars: $100 \mu \mathrm{m}$. (B) $\mu \mathrm{CT}$ analysis of cortical bone at the femoral midshaft of virgin and lactating Cts $k^{f \mid / f l}$ mice (black dots and squares, respectively) and Ctsk $k^{\text {ocy }}$ mice (red dots and squares, respectively) ( $n=7-9$ per group). (C) $\mu \mathrm{CT}$ analysis of trabecular bone at the distal femur of virgin and lactating Ctsk $^{f / / f l}$ (black dots and squares, respectively) and Ctsk ${ }^{\circ c y}$ (red dots and squares, respectively) mice ( $n=7-9$ per group). Results represent the mean \pm SEM. ${ }^{\dagger} P<0.05$ versus virgin $C t s k^{f l / f 1}$ mice; ${ }^{\ddagger} P<0.05$ versus virgin $C t s k^{0 c y}$ mice; and ${ }^{\#} P<0.05$ versus lactating $C$ ts $^{f l / f l}$ mice; 2-way ANOVA followed by Fisher's PLSD test.

Deletion of Ctsk in osteocytes prevents the lactation-induced decrease in serum PTH, maintaining high $1,25(\mathrm{OH})_{2} \mathrm{D}$ levels and ensuring normal serum calcium. Since bone remodeling and calcium metabolism are also affected by hormonal changes during lactation, we next explored the effects of deletion of Ctsk in osteocytes on hormonal regulation. During lactation, and in addition to the increase in PthrP mRNA expression in osteocytes that we observed (Figure 6A), the mammary gland produces PTHrP to mobilize calcium, in part from the skeleton, for milk production $(20,40)$. As a consequence of the circulating levels of PTHrP, PTH levels decrease, and, in mice, $1,25(\mathrm{OH})_{2} \mathrm{D}$ levels increase to maintain appropriate serum calcium levels via intestinal absorption in addition to calcium mobilized from bone $(20,41)$. To determine how osteocyte Ctsk affects these regulatory mechanisms, we measured serum calcium, PTH, and $1,25(\mathrm{OH})_{2} \mathrm{D}$, and PTHrP plasma levels in lactating $C t s k^{o c y}$ and control mice. As expected, serum calcium was maintained

erties of femurs in the same animals that were studied by $\mu \mathrm{CT}$, histomorphometry, and cortical bone gene expression analyses. In nonlactating controls, we found that deletion of Ctsk in osteocytes did not influence bone mechanical properties with the exception of yield load (Figure 8 and Supplemental Table 7). Lactating mice of both genotypes had significantly lower maximum bending moment, bending stiffness, and estimated strength than did the corresponding control virgin mice, but deletion of Ctsk in osteocytes partially but significantly protected the mice from the negative effects of lactation: maximum bending moment and failure moment were significantly greater in $C t s k^{o c y}$ lactating mice (Figure 8 and Supplemental Table 7). Although several other parameters showed some degree of protection from the negative effects of lactation, these differences were not significant. within the normal range in both lactating control mice and lactating Ctsk $^{o c y}$ mice (Supplemental Table 5). In contrast, serum PTH levels were decreased in lactating control mice (Figure 5 and Supplemental Table 5), but deletion of Ctsk in osteocytes prevented this decrease, maintaining PTH levels in Ctskocy lactating mice within the normal, nonlactating range (Figure 5 and Supplemental Table 5). We observed the expected increase in PTHrP plasma levels in lactating control mice, whereas in $C t s k^{o c y}$ mice, only a trend toward an increase was observed (Figure 5 and Supplemental Table 5). In contrast, $1,25(\mathrm{OH})_{2} \mathrm{D}$ levels were markedly and significantly increased in both lactating control and $C t s k^{o c y}$ mice (Figure 5 and Supplemental Table 5). Noticeably, however, $1,25(\mathrm{OH})_{2} \mathrm{D}$ levels were significantly higher in lactating control and Ctsk ${ }^{o c y}$ mice compared with levels in virgin mice (Figure 5 and Supplemental Table 5). 

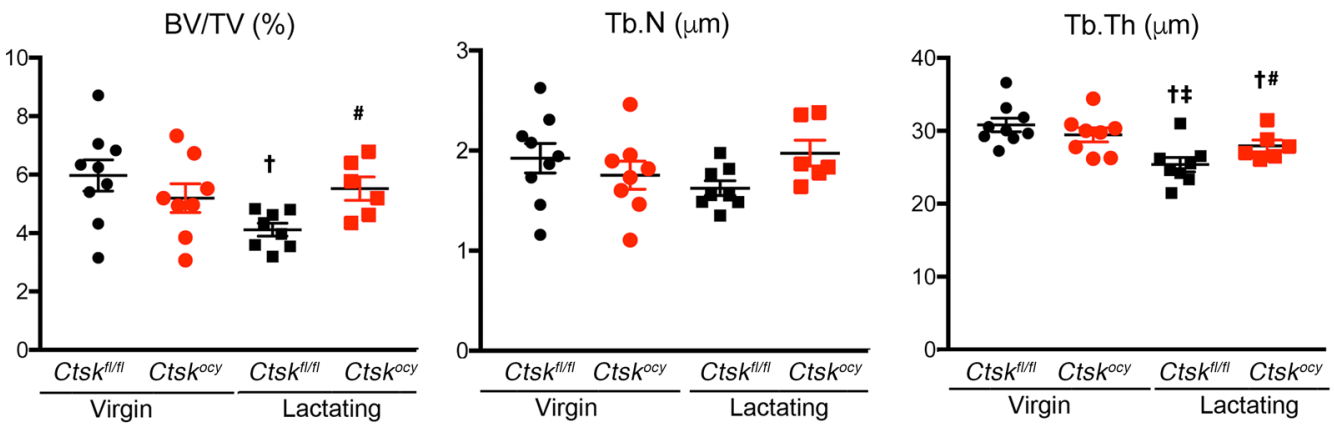

Figure 4. Osteocyte-specific deletion of Ctsk prevents bone loss in lactating mice. Histomorphometric analysis of distal femurs from virgin and lactating Ctsk $^{f \mid / f l}$ (black dots and squares, respectively) and Ctsk ${ }^{\text {ccy }}$ (red dots and squares, respectively) mice. Results represent the mean \pm SEM. ${ }^{\dagger} P<0.05$ versus virgin Ctsk ${ }^{f l / f l}$ mice; ${ }^{\ddagger} P<0.05$ versus virgin Ctsk ${ }^{\text {ocy }}$ mice; and ${ }^{\#} P<0.05$ versus lactating Ctsk $^{f l / f l}$ mice $(n=$
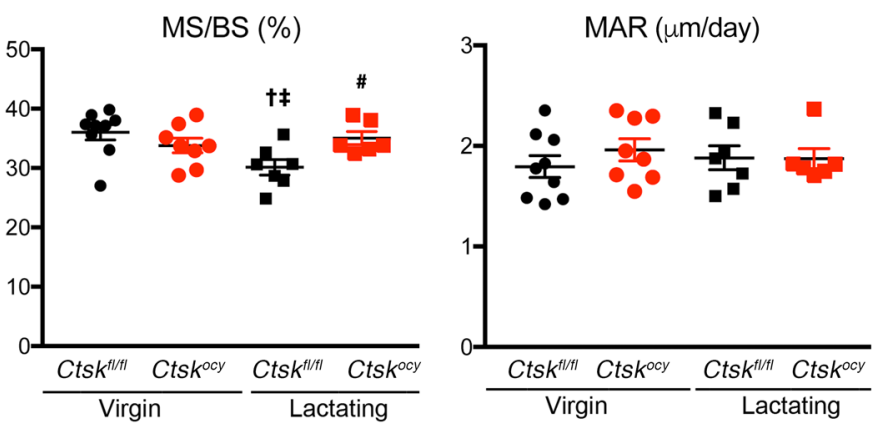

BFR/BS ( $\mu \mathrm{m}^{3} / u \mathrm{~m}^{2} /$ day $)$

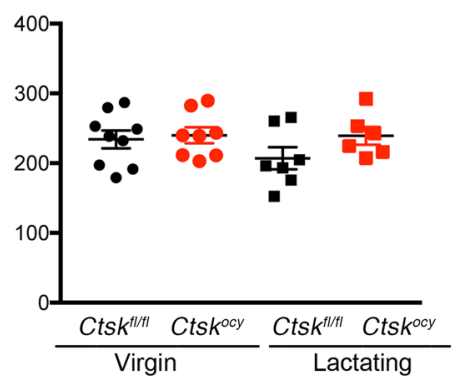
6-9 per group). A 2-way ANOVA followed by Fisher's PLSD test was used to determine statistical significance. 0.Th, osteoid thickness.

Ob.S/BS (\%)

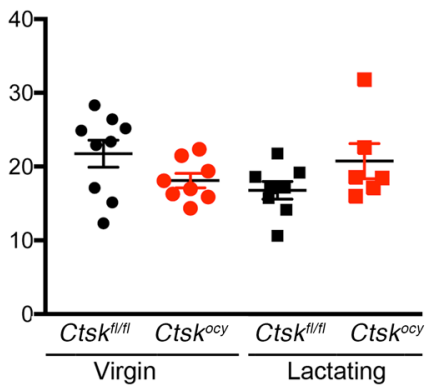

Oc.S/Bs (\%)

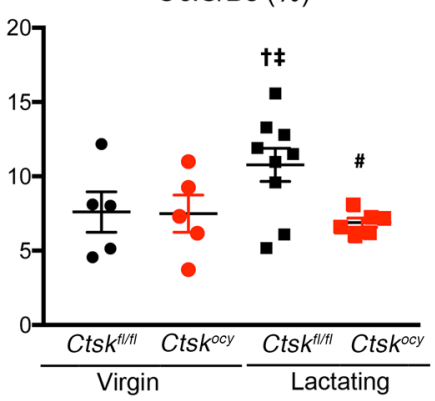

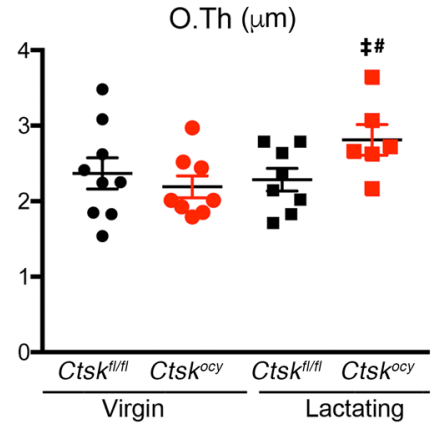

Deletion of Ctsk in osteocytes does not affect milk calcium or skeletal development and density in the offsprings of lactating Ctsk ${ }^{\text {ocy }}$ mothers. Given that these hormonal changes ensured that serum calcium was maintained in the normal range, we then asked whether calcium concentration in the milk is also maintained and whether an appropriate supply of calcium from Ctsk ${ }^{o c y}$ mothers to the offspring skeleton is ensured. For this purpose, we measured calcium content in milk and in the pup's skeleton using $\mu \mathrm{CT}$. We observed no changes in milk calcium content ( $2.19 \pm 0.15$ vs. 2.28 $\pm 0.32 \mathrm{mg} / \mathrm{mg}$ protein) and no differences in cancellous bone volume between the offspring of control and Ctsk ${ }^{o c y}$ females (Figure 9 and Supplemental Table 5), indicating that, despite the decrease in osteocytic perilacunar resorption and the low osteoclast numbers and serum CTX1 levels, deletion of Ctsk in osteocytes had no effect on net calcium transfer via the milk to the pups and therefore on their bone development or homeostasis.

\section{Discussion}

The skeleton is a storehouse for minerals, including calcium, which is mobilized in situations of high calcium demand such as lactation $(20,40)$. Lactation induces a large transfer of bone calcium to the circulation of lactating mothers to allow for the proper amount of calcium in milk, a physiological process that induces significant bone loss (40). A well-defined mechanism by which bone loss occurs during lactation involves the upregulation of osteoclastogenesis and osteoclast-dependent bone resorption (42). However, recent studies have confirmed that osteocytes can remodel their perilacunar space and resorb the perilacunar matrix, enlarging their lacunae during lactation (15, 17, 18, 27, 36) and potentially contributing to calcium mobilization from the skeleton. Indeed, the total osteocyte lacunar surface area has been estimated to be at least 10 times that of the trabecular bone surfaces upon which osteoclasts can act $(11,12,43)$. Hence, small changes in osteocyte lacunae can mean large changes in mineral content at the level of the whole animal or human. On the one hand, the importance of perilacunar resorption (osteocytic osteolysis) in ensuring calcium mobilization from the skeleton, especially at times of high calcium demand such as during lactation, is still controversial. On the other hand, the influence of the matrix degradation that occurs during perilacunar resorption on the regulation of osteocyte behavior, bone remodeling and skeletal homeostasis is only beginning to be explored $(17,27,36)$. In this study, we show that osteocyte-derived Ctsk plays a key role in the mechanism by which osteocytes regulate not only the size 

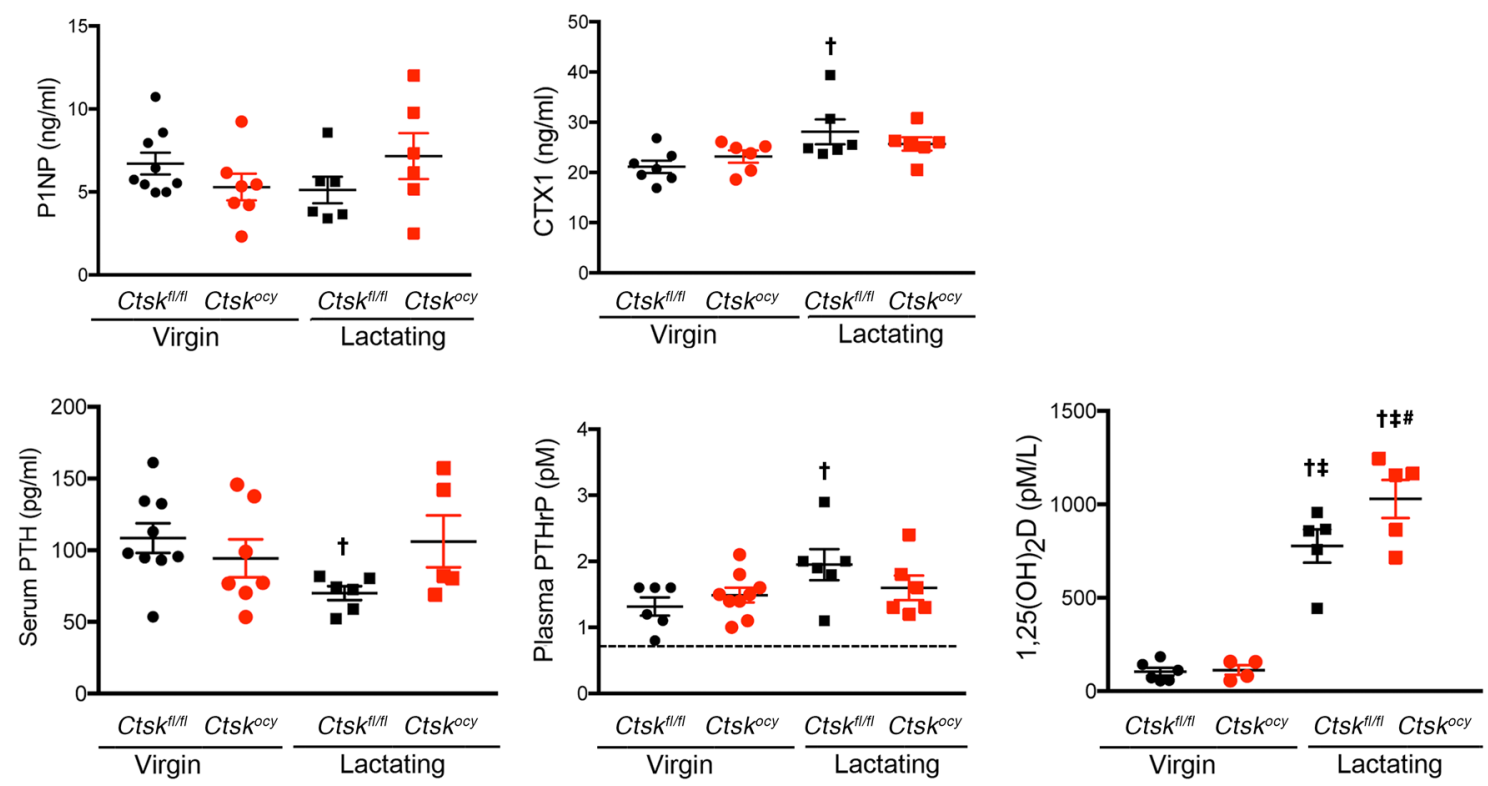

Figure 5. Deletion of Ctsk in osteocytes affects serum hormone and CTX1 levels. P1NP, CTX1 PTH, PTHrP, and 1,25(OH) ${ }_{2}$ vitamin $\mathrm{D}_{3}$ levels in serum or plasma from virgin and lactating Ctsk $k^{f l f l}$ (black dots and squares, respectively) and Ctsk ${ }^{o c y}$ (red dots and squares, respectively) mice. For PTHrP, the dotted line represents the limit of detection of the assay. Data are expressed as the mean \pm SEM ( $n=5$-9 per group). ${ }^{\dagger} P<0.05$ compared with virgin $C t s k^{f / / f l}$ mice; ${ }^{\ddagger} P<$ 0.05 versus virgin Ctsk ${ }^{\circ c y}$ mice; ${ }^{\#} P<0.05$ versus lactating $C t s k^{f l / f l}$ mice; 2 -way ANOVA followed by Fisher's PLSD test.

of their lacunae but also their own gene expression profile, affecting the regulation of skeletal homeostasis and calcium metabolism in response to lactation (Figure 10). Indeed, our results show that Ctsk-dependent degradation of its surrounding matrix by the osteocyte is required not only for perilacunar resorption and enlargement of osteocyte lacunae, but also for the induction of osteoclast differentiation, which ultimately lead to bone loss and fragility. Consequently, preventing matrix degradation around osteocytes by the targeted deletion of Ctsk decreases not only perilacunar resorption, but also prevents the increase in osteoclast numbers and the mild decrease in bone formation, bone loss and, in part, the increase in bone fragility that normally occur during lactation in mice. This was due in part to a lower Rankl/ Opg ratio in osteocytes in the absence of Ctsk, in the presence of low levels of Sost. The inability of Ctsk-deleted osteocytes to efficiently enlarge their lacunae and generate the appropriate signals to increase osteoclast numbers would, if not compensated by other mechanisms, be expected to at least partially impair calcium mobilization from bone. In lactating women, suppression of serum PTH is usually observed (42) as a consequence of the high calcium levels induced by mammary gland-derived PTHrP (40). We show here that the same regulatory phenomenon occurs in control lactating mice when they are not simultaneously subjected to a low-calcium diet, as done in many previously published reports $(19,36)$. Here, however, PTH levels failed to decrease in Ctsk ${ }^{o c y}$ lactating mice, indicating that the negative feedback loop that normally downregulates PTH during lactation is impaired in the absence of Ctsk in osteocytes, and this occurs despite the high mRNA levels of PthrP in the bones of Ctskocy lactating mice. We interpret these data as showing that calcium mobilization from bone is indeed impaired in the absence of Ctsk in osteocytes but that this impairment is compensated by significant hormonal changes. Instead of being partially suppressed in control lactating mice, we found that the parathyroid gland continued to secrete PTH during lactation in Ctsk ${ }^{o c y}$ mice in order to maintain serum calcium levels. However, in the absence of Ctsk, osteocytic osteolysis and osteoclastogenesis failed to respond appropriately to the demand for calcium. Consequently, this process required not only higher PTH levels but also higher $1,25(\mathrm{OH}){ }_{2} \mathrm{D}$ as well as higher local PTHrP levels to maintain serum and milk calcium levels in the normal range (Figure 10). Together, PTH and PTHrP, and possibly the low levels of bone $F g f 23$, induced by high $1,25(\mathrm{OH})_{2} \mathrm{D}$ (44), allowed sufficiently effective intestinal calcium absorption in these mice that were not deprived of dietary calcium. As shown by others, $1,25(\mathrm{OH})_{2} \mathrm{D}$ protects mice from bone loss during lactation (45). The higher levels of circulating PTH and 1,25(OH) ${ }_{2} \mathrm{D}$ in $C t s k^{o c y}$ versus control lactating mice, together with the high levels of PthrP in bone itself, may also explain the limited increase in circulating PTHrP levels we detected in the lactating Ctsk ${ }^{o c y}$ mice. Altogether, this mechanism ensured the maintenance of sufficient calcium levels in the maternal serum and in milk, allowing normal skeletal development of the offspring of Ctsk ${ }^{o c y}$ mothers, despite the prevention of perilacunar resorption and osteoclast recruitment. Most important, this led to the prevention of bone loss in $C t s k^{o c y}$ mothers, while sufficient calcium was provided to the offspring. In contrast, as shown by Ardeshirpour et al. (46), when the mother is deprived of dietary calcium in addition to a blockage of osteoclastogenesis, maternal calcium, PTH, and pup growth are affected. Together, these findings further establish that in mice, in which the demand for calcium is high, bone resorption, whether by osteocytes or osteoclasts, and dietary calcium absorption back each other up to ensure proper levels of calcium in the milk (42). Although our study clearly establishes these links in lactating mice, it should be noted that in humans, 
A

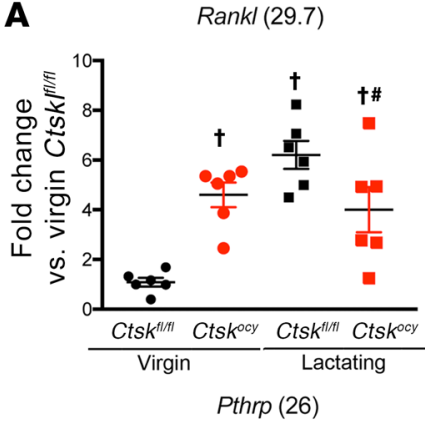

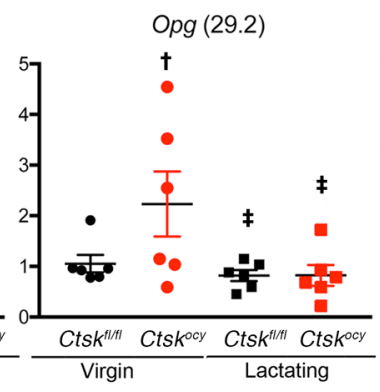

Mmp13 (24.7)

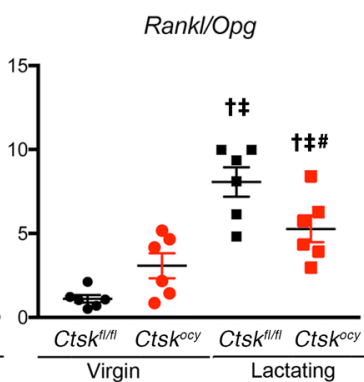

Sost (23.5)
Ctsk (24.6)

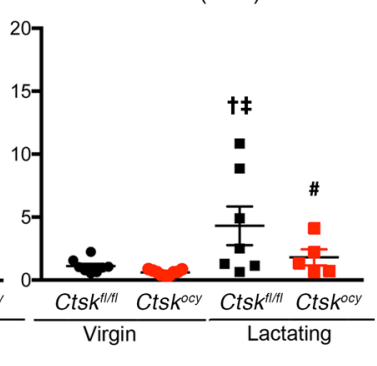

Fgf23 (33)
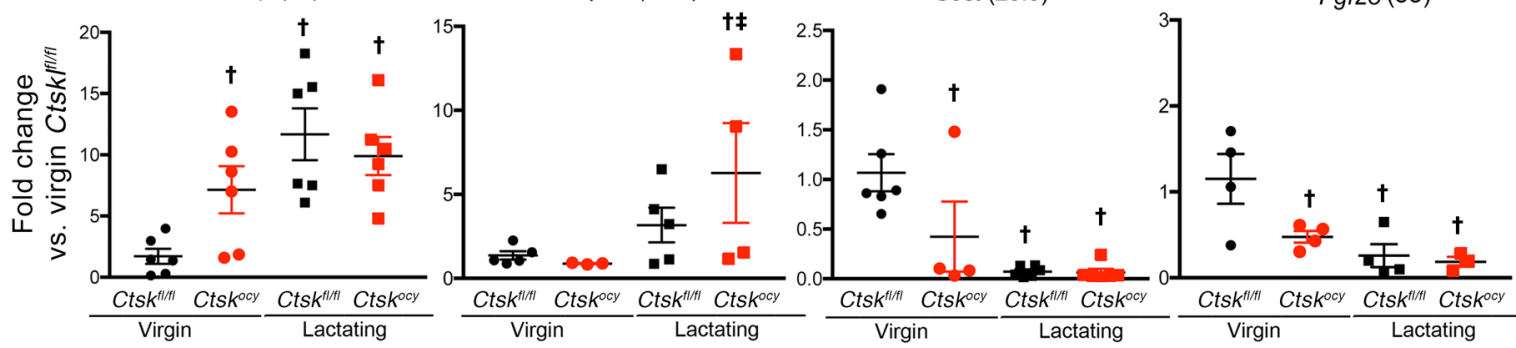

B

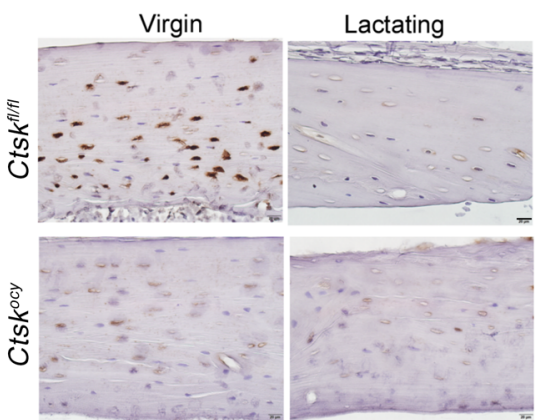

C

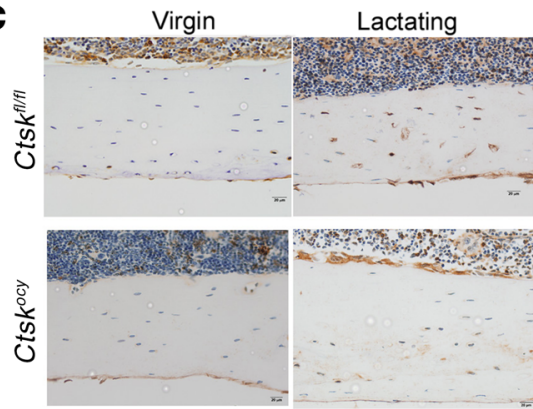

D

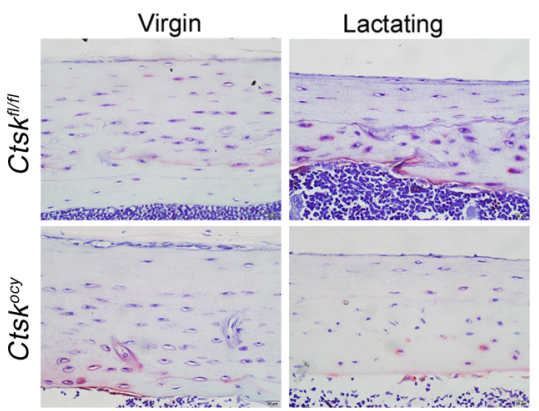

D

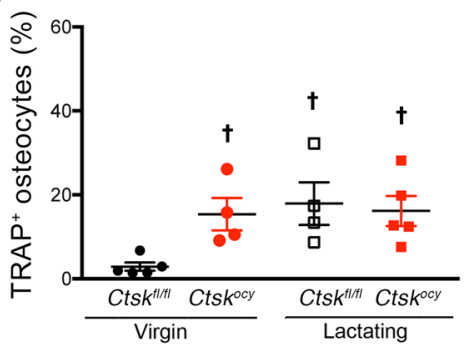

Figure 6. Deletion of Ctsk in osteocytes affects the expression of genes involved in bone remodeling. (A) qRT-PCR analysis of mRNA expression in BM-depleted tibiae and femurs from virgin and lactating Ctsk $k^{f \mid f l}$ (black dots and squares, respectively) and Ctsk ${ }^{\circ c y}$ (red dots and squares, respectively) mice ( $n=5-9$ per group). Numbers in parentheses are the Ct values for the highest level of expression of each gene. (B-D). Representative images of sclerostin (B), Ctsk immunostaining (C), and TRAP staining (D) in osteocytes from virgin and lactating Ctsk ${ }^{f \mid l f l}$ and Ctsk ${ }^{\circ c y}$ mice ( $n=4-7$ per group). Scale bars: $20 \mu \mathrm{m}$. (E) Quantification of TRAP staining ( $n=4$ to 5 per group). Data are expressed as the mean \pm SEM. ${ }^{\dagger} P<0.05$ versus virgin $C$ ts $k^{f l f f l}$ mice; ${ }^{\ddagger} P<0.05$ versus virgin Ctsk $k^{\text {ocy }}$ mice; and ${ }^{\#} P<0.05$ versus lactating Ctsk ${ }^{f l / f l}$ mice; 2 -way ANOVA followed by Fisher's PLSD test.

$1,25(\mathrm{OH})_{2} \mathrm{D}$ levels and calcium absorption have been reported to be unaltered in lactating mothers $(42,47)$. Indeed, randomized interventional trials and observational studies have shown that women generally maintain the same degree of skeletal resorption during lactation, regardless of low or high calcium intake, suggesting that dietary calcium absorption is not as crucial in lactating women as it is in mice $(20,42)$.

Thus, perilacunar resorption and matrix degradation around the osteocyte are an integral part of the response of osteocytes to the increase in PTHrP (18) and 1,25(OH) ${ }_{2} \mathrm{D}(48)$ that occurs during lactation. However, perilacunar resorption and matrix degradation appear to be required for more than just lacunar enlargement, and our results suggest that these are required steps for PTH and PTHrP to induce an increase in osteoclast numbers (Figure 10). Indeed, as observed previously with global or osteoclast-targeted deletion of Ctsk (4-6), Rankl expression and the Rankl/Opg ratio were increased in osteocytes of Ctsk ${ }^{o c y}$ mice at steady state, independent of lactation. During lactation, Rankl and the Rankl/Opg ratio were, 
A

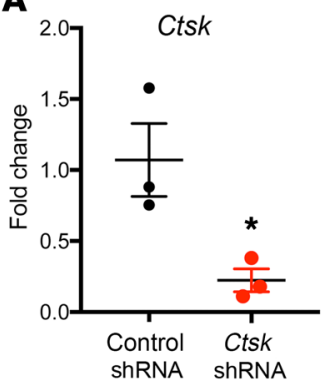

B

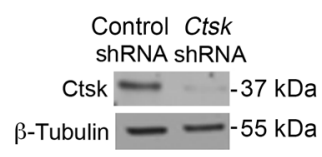

C

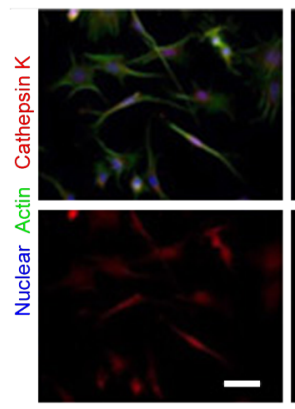

Opg

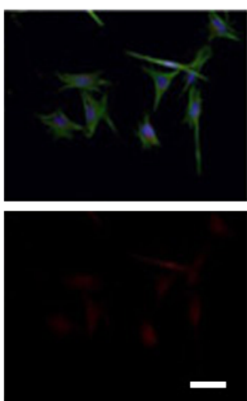

D

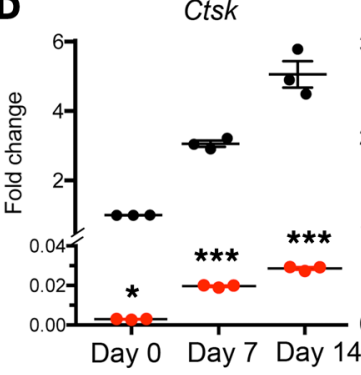

Rankl

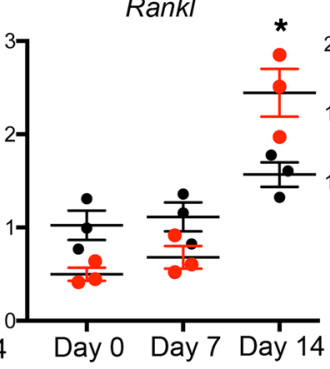

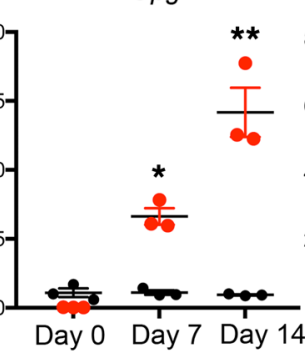

Sost

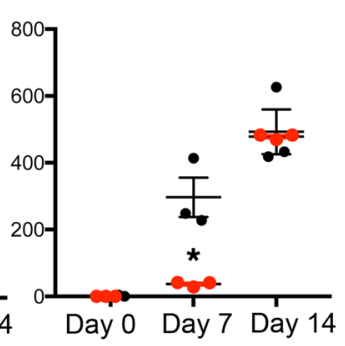

Figure 7. Ctsk silencing in Ocy454 cells affects the expression of genes involved in bone remodeling. (A) Efficiency of Ctsk deletion in Ocy454 cells. Control shRNA (black dots) and Ctsk shRNA (red dots) (catalog TRCN0000054624). Data shown represent the mean of triplicate experiments \pm SEM and were normalized to the expression of 185 mRNA $(n=3)$. ${ }^{*} P<0.05$ by Student's 2 -tailed $t$ test. (B) Densitometric analysis of Western blotting showing the efficiency of Ctsk silencing. $\beta$-Tubulin was used as a loading control $(n=3)$. (C) Ctsk immunostaining in control shRNA and Ctsk shRNA Ocy454 cells showing Ctsk (red), actin (green) and nucleus (blue) $(n=3)$. Scale bars: $20 \mu \mathrm{m}$. (D) qRT-PCR analysis of mRNA expression in control shRNA (black dots) and Ctsk shRNA (red dots) Ocy454 cells. Data shown are the mean of triplicate experiments \pm SEM and were normalized to the expression of $18 \mathrm{~S}$ mRNA ( $n=3$ ). ${ }^{*} P<0.05,{ }^{* *} P<0.01$, and ${ }^{* *} P<0.001$, by Student's $t$ test for comparisons of control shRNA with Ctsk shRNA at each time point.

however, markedly increased in control mice but remained at the same, albeit high, levels in the absence of Ctsk. This suggests that the inability of osteocytes to efficiently induce osteoclastogenesis when depleted of Ctsk cannot be simply attributed to a lack of Rankl or a low Rankl/Opg ratio. Instead, these findings suggest that matrix degradation by osteocytes is dependent on Ctsk in osteocytes and that this process initiates a feedback-signaling loop that regulates osteoclastogenesis.

How can Ctsk-dependent matrix degradation by osteocytes contribute to the regulation of osteocytic genes that in turn regulate bone remodeling? Although the present study does not provide a definitive answer to this question, it clearly demonstrates that gene expression is altered in osteocytes after targeted Ctsk deletion. Our study showed that Rankl and Opg mRNA levels, as well as TRAP activity, were increased in nonlactating $C t s k^{\text {ocy }}$ osteocytes relative to control mice, whereas Sost and Fgf23 mRNA levels were decreased, consistent with the increase in PthrP expression by osteocytes in Ctsk-deleted osteocytes (38), independent of lactation. Mechanistically, we hypothesize that the absence of Ctsk could prevent the degradation of products from the matrix, allowing these products to be present in the osteocyte lacunar microenvironment at high enough concentrations to signal back to receptors on the osteocyte membrane. Alternatively, lack of Ctsk may prevent the activation of factors such as TGF- $\beta$. According to this hypothesis, the osteocyte would induce matrix degradationdependent signaling cascades via mobilized and/or activated matrix-derived growth factors, such as TGF- $\beta$ or insulin growth factor 1 (IGF1), and/or proteolytically cleaved fragments of matrix components, such as collagen. In fact, these molecules have already been implicated in the regulation of osteocytes in vitro and, more recently for MMP13 and TGF- $\beta$ signaling, in vivo (13, $17,49)$. Since it has been shown that Ctsk can fully degrade TGF- $\beta$ (50) as well as IGF1 (51), Ctsk deletion in osteocytes could simply prevent the complete degradation of these growth factors once perilacunar resorption is initiated, allowing activation of signaling from the osteocyte membrane. Alternatively, similar to some other proteolytic enzymes (52-54), Ctsk could bind to cell-surface receptors such as LDL receptor-related protein 1 (LRP1) (52-54) once secreted into the perilacunar space and act as an autocrine ligand. Further work will be required to test these hypotheses and identify whether matrix components and/or Ctsk itself acting as a ligand are critical for allowing osteocytes to generate and transmit the signals required for recruitment of osteoclast precursors and regulation of bone remodeling.

\section{Methods}

Blinding. To avoid bias, data from all studies were collected in a blinded fashion, in which the observer was unaware of the experimental group. Key studies were performed by more than 1 individual to determine whether there was consistency in the observations.

Mice. Generation of the Ctsk $k^{f / f l}$ mice was previously described $(4,6)$, and the 9.6KbDmp1-Cre mice were provided by J.Q. Feng (Department of Biomedical Sciences, Texas A\&M University Baylor College of Dentistry, Dallas, Texas, USA) (55). Both mouse models were on a C57BL/6J background. Mice hemizygous for Dmp1Cre were crossed with $\mathrm{Ctsk}^{f / f l}$ mice to generate Dmp1-Cre Ctsk $\mathrm{C}^{\mathrm{fl} /+}$ 

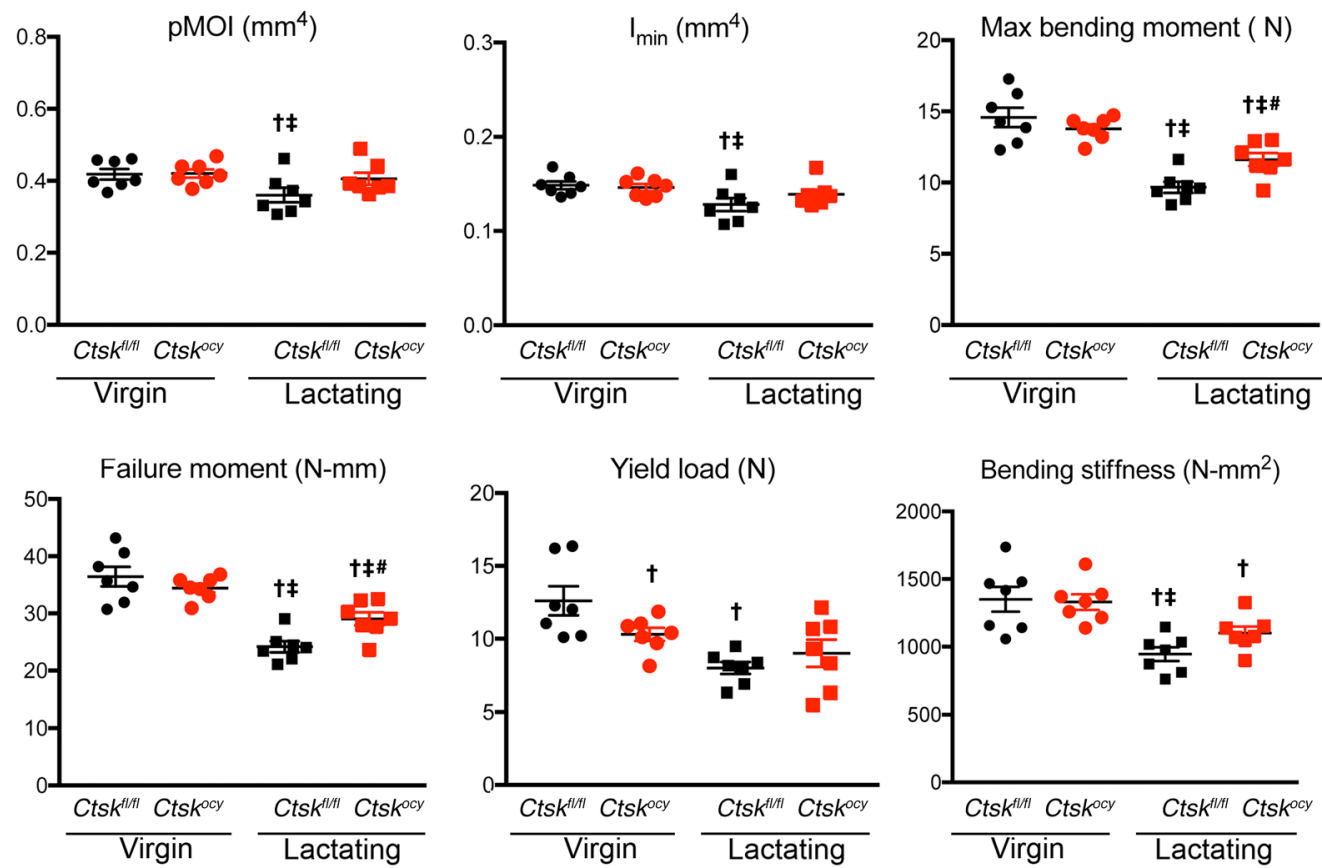

Bending stiffness $\left(\mathrm{N}-\mathrm{mm}^{2}\right)$
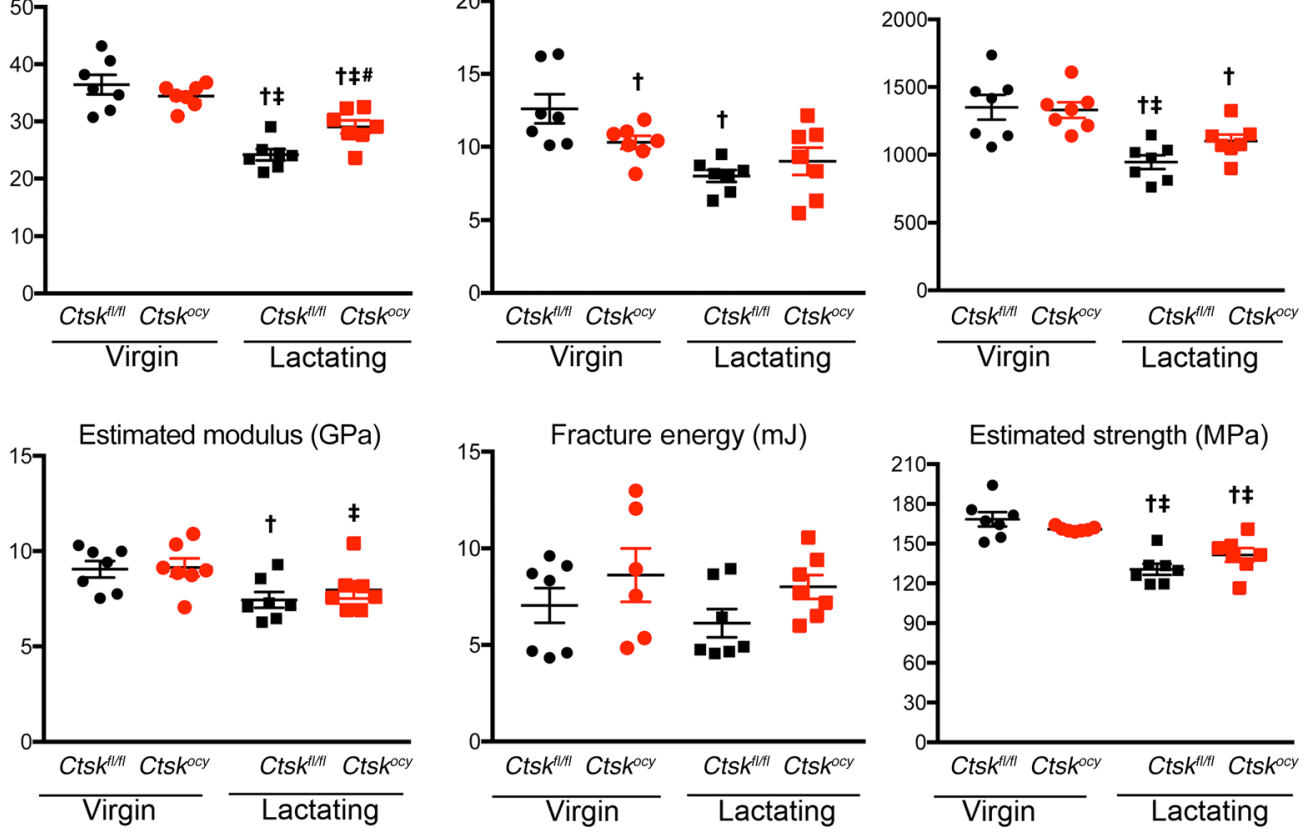

Figure 8. Deletion of Ctsk in osteocytes prevents bone fragility. Three-point bending analysis of femurs from virgin and lactating Ctsk ${ }^{f \mid f f}$ (black dots and squares, respectively) and Ctsk $k^{o c y}$ (red dots and squares, respectively) mice. Results represent the mean $\pm \mathrm{SEM}$. ${ }^{\dagger} P<0.05$ versus virgin $C t s k^{f / f f}$ mice; ${ }^{\ddagger} P<0.05$ versus virgin Ctsk ${ }^{o c y}$ mice; and $\#<0.05$ versus lactating Ctsk ${ }^{f / f /}$ mice; 2-way ANOVA followed by Fisher's PLSD test comparing all groups ( $n=6-7$ per group). N, Newtons.

mice, which were in turn crossed with $C t s k^{f / f l}$ mice to generate Dmp1-Cre Ctsk ${ }^{f / f l}$ mice (referred to herein as Ctsk ${ }^{o c y}$ mice). Ctsk $k^{f / f l}$ mice were then crossed with $C t s k^{o c y}$ mice to obtain Ctsk ${ }^{o c y}$ mice and $\mathrm{Ctsk}^{f / f l}$ littermates, which were used as controls. For lactation studies, 7-week-old Ctsk ${ }^{f / f l}$ and Ctsk ${ }^{o c y}$ female mice were crossed with male mice. After delivery, the litter size was adjusted to 6 to 7 pups to maintain similar suckling intensities. Females were sacrificed on the 12th day of lactation and were approximately 12 weeks old. Offspring of the Ctsk ${ }^{o c y}$ mice and $C t s k^{f / f l}$ littermates were also sacrificed on day 12 for skeletal phenotype analysis. The genotype was determined by sequencing genomic DNA from $C t s k^{o c y}$ and $C t s k^{f / f l}$ mice using forward 5'-CGCGGTCTGGCAGTAAAAACTATC-3' and reverse 5'-CCCACCGTCAGTACGTGAGATATC-3' primers to sequence the Cre transgene and forward $5^{\prime}$-GGAAACTGAGGCACAGAGTTCAG-3' and reverse 5'-GGGGTGTGCACCTATGTGTGTA-3' primers to sequence the floxed allele. Primers against chromosome $\mathrm{Y}$ were used to identify the sex of the pups. All mice were fed a regular diet.

Backscatter scanning electron microscopy. The backscatter scanning electron microscopy (BSEM) imaging technique was used to quantify osteocyte lacunae cortical bone at the mid-diaphysis of femurs. Freshly dissected femurs were embedded in methyl methacrylate and sectioned and the blocks sequentially polished with sandpaper and carbon coated. Images of osteocyte lacunae in cortical bone at the diaphysis were captured at $\times 500$ magnification using BSEM (Zeiss Supra 55) in a standard 0.1- $\mathrm{mm}^{2}$ cortical area 5-6 mm from the proximal growth plate. The "single osteocyte lacunar area" represents the mean of all ( $>100)$ individual osteocyte lacunae measurements within the predefined area. The osteocyte lacunar area, osteocyte perimeter, and osteocyte numbers were quantified using Fiji software in a blinded fashion. Analyses of bones from 5 to 7 mice per group were performed.

$\mu C T$. $\mu \mathrm{CT}$ analysis was performed using a desktop $\mu \mathrm{CT} 35$ (Scanco Medical) according to recommended guidelines (56). Briefly, cortical bone parameters at the femoral midshaft and cancellous bone microarchitecture in the distal femoral metaphysis were determined using a $7-\mu \mathrm{m}$ isotropic voxel size. Eighty-six transverse $\mu \mathrm{CT}$ slices of cortical bone were evaluated, and total cross-sectional volume (millimeter cubed), cortical volume (millimeter cubed), marrow volume (millimeter cubed), and cortical thickness (millimeter) were assessed. For cancellous bone, bone volume fraction (bone volume per tis- 
A

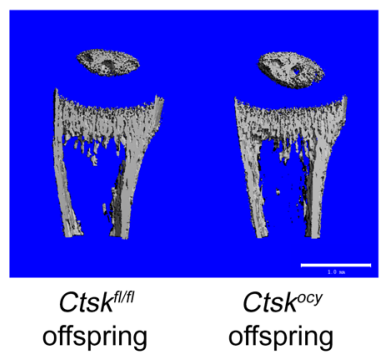

B

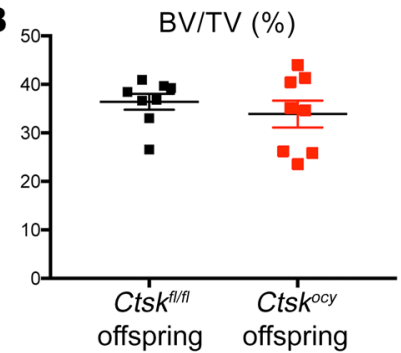

$\mathrm{BMD}\left(\mathrm{mgHA} / \mathrm{cm}^{3}\right)$

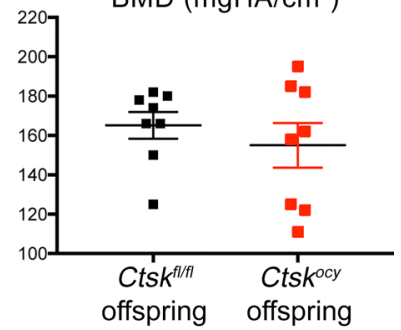

Figure 9. Deletion of Ctsk in osteocytes does not affect offspring skeletal homeostasis. (A) Representative $\mu \mathrm{CT}$ images of the proximal tibia from 12-dayold Cts $k^{f \mid / f l}$ female offspring of lactating $C t s k^{f / f f l}$ and $C t s k^{o c y}$ mice. Scale bar: $1 \mathrm{~mm}$. (B) $\mu \mathrm{CT}$ analysis of BV/TV and bone mineral density (BMD) bones from offspring of lactating Ctsk $k^{f l / f l}$ (black squares) and Ctsk $k^{\text {ocy }}$ (red squares) mice ( $n=8$ per group).

sue volume [BV/TV]) (percentage), trabecular number (Tb.N) (per millimeter), trabecular thickness (Tb.Th) (millimeter), trabecular separation (Tb.Sp) (millimeter), connectivity density (ConnD) (per millimeter cubed), and structural model index (SMI) were analyzed. Analyses of bones from 7 to 9 mice per group were performed. For the analysis of offspring bones, 8 female $\mathrm{Ctsk}^{f / f l}$ pups were subjected to $\mu \mathrm{CT}$ analysis. Intracortical bone volume fraction was measured in a $600-\mu \mathrm{m}$-long region (100 transverse slices) beginning at the top of the proximal tibia metaphysis and extending distally. A threshold of 200 milligram hydroxyapatite per cubic centimeter $\left(\mathrm{mgHA} / \mathrm{cm}^{3}\right)$ was used to segment bone from soft tissue.

Histomorphometry. Twelve-week-old Ctsk ${ }^{f / f l}$ and Ctsk $^{o c y}$ mice were subcutaneously injected with $20 \mathrm{mg} /$ $\mathrm{kg}$ calcein (MilliporeSigma) and demeclocycline (MilliporeSigma) on days 7 and 2 before necropsy, respectively. For lactation studies, virgin and lactating $C t s k^{f / f l}$ and $C t s k^{o c y}$ mice were injected with fluorochrome on days 7 and 2 before necropsy. For phenotype analyses, femurs were fixed in $70 \%$ alcohol and embedded in methylmethacrylate. Toluidine blue and von Kossa staining was performed using $5-\mu \mathrm{m}$ longitudinal sections. Histomorphometric measurements were carried out semiautomatically with the OsteoMeasure image analyzer (OsteoMetric). A standard sampling site with an area of $2.5 \mathrm{~mm}^{2}$ was established in the secondary spongiosa of the distal metaphysis. All parameters were expressed according to standardized nomenclature $(57,58)$. Dynamic parameters including the mineralizing surface per bone surface (MS/ $\mathrm{BS})$ (percentage), the mineral apposition rate (MAR) (micrometer per day), and the bone formation rate expressed per bone surface (BFR/BS) (micrometer cubed per micrometer squared per year) and per bone volume (BFR/BV) (percentage per year) were quantified. Static parameters including BV/TV (percentage), Tb.Th (micrometer), Tb.Sp (micrometer), and Tb.N (per millimeter), osteoblast surface per bone surface (Ob.S/BS) (percentage), osteoblast number per bone perimeter (N.Ob/B.Pm) (per millimeter), osteoclast surface per bone surface (Oc.S/BS) (percentage), and osteoclast number per bone perimeter (N.Oc/B.Pm) (per millimeter) were measured. Analyses of bones from 6 to 9 mice per group were performed.
Cell culture. Ocy 454 osteocyte cells were plated at $1 \times 10^{5}$ cells/ $\mathrm{ml}$ and allowed to reach confluence at $33^{\circ} \mathrm{C}$ in $\alpha$-MEM supplemented with $10 \%$ FBS and $1 \%$ Anti-Anti (Life Technologies, Thermo Fisher Scientific) to induce proliferation as previously described (39). Subsequently, cells were transferred to $37^{\circ} \mathrm{C}$ and differentiated for the indicated durations. Ocy 454 cells were infected with 2 distinct lentivirus particles expressing shRNAs targeting Ctsk (TRCN0000054624 and TRCN0000030591, Mission TRC, MilliporeSigma) for 24 hours, followed by puromycin $(10 \mu \mathrm{g} / \mathrm{ml})$ selection at $33^{\circ} \mathrm{C}$. A nontargeted shRNA (Non-target/Control-SHC002,
A

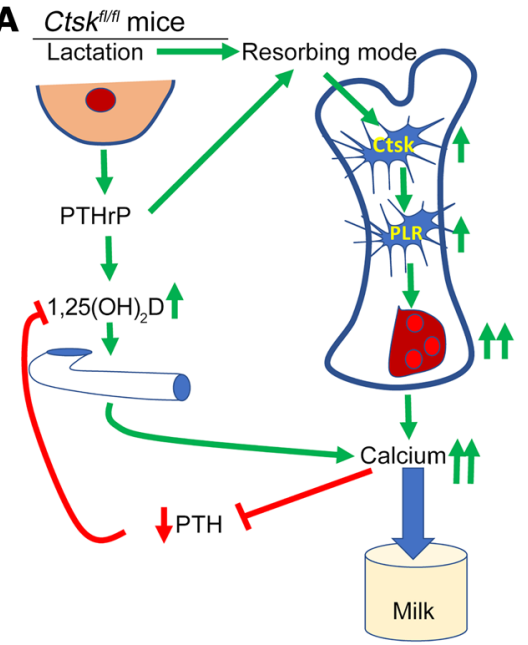

B Ctsk $k^{t / f t}$ mice

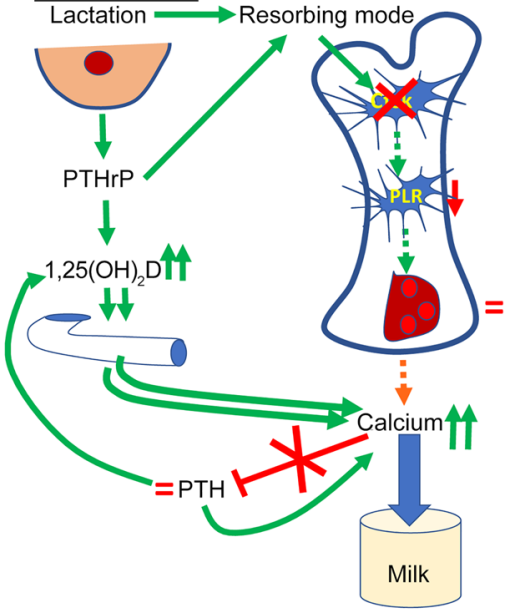

Figure 10. Schematic representation of the relationship between osteocytic osteolysis, bone remodeling, and calcium metabolism during lactation in mice in the presence or absence of Ctsk in osteocytes. (A) In the presence of Ctsk in osteocytes (control lactating mice), the changes induced by the lactating mammary gland, including increased secretion of PTHrP, switch the osteocytes to the resorbing mode, increasing TRAP activity and Ctsk expression and leading to increased perilacunar resorption (PLR) and lacunar enlargement. These changes in the osteocyte microenvironment lead to an increase in PTHrP and the Rankl/Opg ratio, thereby increasing osteoclastogenesis, bone resorption, and serum calcium. This increase in serum calcium is also secondary to higher PTHrP and 1,25(OH) $\mathrm{D}$ acting on the kidney and the gut, resulting in both calcium supply to the milk and repression of endogenous PTH secretion. (B) In the absence of Ctsk in osteocytes (Ctsk ${ }^{\text {ocy }}$ lactating mice), although the changes induced in the mammary gland by lactation increase TRAP activity and Pthrp in osteocytes, deletion of Ctsk prevents PLR, enlargement of the lacuna, and an increase in the Rankl/Opg ratio and osteoclastogenesis. The lack of osteocytic osteolysis and osteoclastogenesis limits the supply of calcium to serum; this is sensed by the parathyroid gland, which continues to secrete PTH at prelactation levels and, together with PTHrP, enhances $1,25(\mathrm{OH})_{2} \mathrm{D}$ levels and thus calcium absorption from the gut, compensating for the limited supply of calcium coming from bone and ensuring appropriate calcium levels in the blood and milk. 
MilliporeSigma) was used to generate control cells. Analyses were performed at least 3 times in duplicate.

Osteoclasts were generated on coverslips. BM cells isolated from $C t s k^{f / f l}$ and $C t s k^{o c y}$ mice were cultured in $\alpha$-MEM containing $10 \% \mathrm{FBS}$, 100 units/ml penicillin, and $100 \mu \mathrm{g} / \mathrm{ml}$ streptomycin for 24 hours to generate BM macrophages (BMMs). BMMs were cultured on coverslips in $\alpha$-MEM for 2 days with macrophage-CSF (M-CSF) $(20 \mathrm{ng} / \mathrm{ml})$, and for an additional 6 days in the same medium with M-CSF (20 ng/ $\mathrm{ml}$ ) and RANKL (3.3 ng/ml) (R\&D Systems). Likewise, for RNA isolation, BMMs were isolated from BM-flushed tibiae and femurs of 6- to 8-week-old Ctsk ${ }^{f / f l}$ and Ctsk ${ }^{o c y}$ mice. For osteoclast generation, cells were cultured with M-CSF and RANKL for 4 days.

Immunohistochemistry. Paraffin-embedded, decalcified tibiae were fixed in $10 \%$ neutral buffered formalin (NBF). Longitudinal sections (5- $\mu \mathrm{m}$-thick) were incubated with antibodies specific to Ctsk (ab19027, Abcam) and sclerostin (AF1589, R\&D Systems) at $4^{\circ} \mathrm{C}$ overnight and then treated with tyramide signal amplificationbiotin (TSA biotin) (PerkinElmer) and streptavidin-HRP according to the manufacturer's instructions. Analyses of bones from 3 to 4 mice were performed. Osteoclasts were fixed with $3.7 \%$ formaldehyde in PBS and permeabilized in $0.05 \%$ saponin followed by acetone. Cells were incubated in anti-Ctsk antibody (ab19027, Abcam) and incubated 24 hours later with fluorescent secondary antibody (Alexa Fluor 488, Green, A-11034, Thermo Fisher Scientific) and mounted in FluorSave (Calbiochem, MilliporeSigma). Nuclei were labeled with TO-PRO-3 (T3605, Thermo Fisher Scientific) in the secondary antibody solution. Ctsk-expressing osteoclasts were visualized using a 510 Meta laser scanning confocal microscope (Carl Zeiss).

RNA extraction, cDNA synthesis, and qRT-PCR. Total RNA was isolated from tibiae and femurs flushed to remove BM and from mammary glands using a monophasic solution of guanidine isothiocyanate and phenol according to the manufacturer's instructions (TRIzol Reagent, Invitrogen, Thermo Fisher Scientific), followed by RNA clean-up using an RNeasy Plus Mini Kit (QIAGEN). RNA from Ocy454 cells and BMM-derived osteoclasts were isolated using the RNeasy Plus Kit (QIAGEN). Total RNA ( $1 \mu \mathrm{g})$ was used to synthesize first-strand cDNA with SuperScript II (Invitrogen, Thermo Fisher Scientific) for reverse transcription PCR. mRNA levels encoding each gene of interest were normalized to actin mRNA in the same sample, and the relative expression of the genes of interest was determined using the formula of Livak and Schmittgen (59). Data are presented as the fold change relative to virgin $C t s k^{f / f l}$ mice, Ocy 454 cells on day 0, Ocy 454 control shRNA, or Ctsk ${ }^{f / f l} \mathrm{BMM}-$ derived osteoclasts. Analyses of bones from 5 to 9 mice per group, mammary glands from 4 to 5 mice per group, and Ocy 454 cells and BMM-derived osteoclasts from 3 mice per group.

Western blot analysis. Protein $(10 \mu \mathrm{g})$ was resolved by SDS-PAGE and transferred electrophoretically onto nitrocellulose membranes using a semi-dry system (Bio-Rad). Immunodetection was performed with anti-Ctsk (ab19027, Abcam) and anti- $\beta$-tubulin (ab6046, Abcam) antibodies. Immunoreactive proteins were visualized using ECL reagents (GE Healthcare). Analyses were performed at least 3 times in duplicate.

TRAP and silver nitrate staining. Paraffin-embedded sections of decalcified tibiae were used for TRAP and silver nitrate staining. For TRAP staining, sections were stained with Fast Red Violet LB
(MilliporeSigma) dissolved in TRAP solution for 15 minutes at $37^{\circ} \mathrm{C}$. TRAP solution consisted of $100 \mathrm{mM}$ sodium acetate, $50 \mathrm{mM}$ sodium tartrate, naphthol AS-MX phosphate, and $N, N$-dimethyl formamide (MilliporeSigma), with adjustment of the $\mathrm{pH}$ to 5.0. Images of $\mathrm{TRAP}^{+}$ osteocytes and TRAP- osteocytes in the diaphysis of cortical bone were acquired using a microscope (Leica MZFLIII Microscope). TRAP $^{+}$osteocytes within 4 and $5 \mathrm{~mm}$ from the proximal end of the femur were quantified in a blinded fashion. For silver nitrate staining, sections were incubated with 2 parts $50 \%$ silver nitrate and 1 part $1 \%$ formic acid with $2 \%$ gelatin solution at room temperature for 55 minutes as previously reported (60). Analyses of bones from 5 to 7 mice were performed.

Biomechanical testing. Left femurs were mechanically tested in 3-point bending using an electrical force materials testing machine (Electroforce 3230, Bose Corporation). The bending fixture had a span length of $10 \mathrm{~mm}$. The test was performed with the load point in displacement control, moving at a rate of $0.03 \mathrm{~mm} / \mathrm{sec}$. All of the bones were positioned in the same orientation during testing, with the cranial surface resting on the supports and loaded in tension. Bending stiffness $\left(\mathrm{N}-\mathrm{mm}^{2}\right)$, estimated modulus of elasticity (gigapascals [GPa]), estimated bending strength (megapascals [MPa]), and fracture energy (millijoules [mJ]) were calculated on the basis of the force and displacement data from the tests and the midshaft geometry measured with $\mu \mathrm{CT}$. Yield was defined as the point at which there was a $10 \%$ reduction in secant stiffness relative to the initial secant stiffness. Fracture energy is the energy that was required to cause the femur to fracture and was calculated by finding the area under the force-displacement curve using the Riemann sum method. Bending stiffness was calculated using the linear portion of the force-displacement curve. The minimum moment of inertia $\left(\mathrm{I}_{\min }\right)$ was used when calculating the estimated modulus of elasticity and bending strength. Polar moment of inertia and post-yield deformation analyses of bones from 6 to 7 mice per group were performed.

Serum and plasma analysis. For biochemical measurements, blood from virgin and lactating $C t s k^{f / f l}$ and $C t s k^{o c y}$ mice was obtained by cheek pouch puncture after a 3-hour fast, and serum was obtained by centrifugation using serum separator tubes. Biochemical measurements were done using commercially available ELISA kits for PTH (Immutopics), and 1,25 $(\mathrm{OH})_{2}$ vitamin $\mathrm{D}_{3}$ (Immunodiagnostic Systems). Serum P1NP and CTX were measured using commercial kits from Immunodiagnostic Systems, according to the manufacturer's instructions. PTHrP plasma levels were measured as previously reported (61). Briefly, blood was collected into commercially available anticoagulant-treated tubes (Thermo Fisher Scientific) containing a protease inhibitor cocktail of aprotinin, leupeptin, and pepstatin. The 2-site human PTHrP immunoradiometric sandwich assay (DSL 8100, Beckman Coulter) was modified by replacing the DSL 8100 antibody-coated tubes with a 96-well plate coated with anti-human PTHrP(1-36), which was affinity-purified from rabbit anti-147 antibody. Standard curves were constructed using the kit standards diluted in mouse plasma that had been incubated overnight at room temperature to degrade endogenous PTHrP. Samples and standards were incubated overnight in the antibody-coated wells at $4^{\circ} \mathrm{C}$, washed, and incubated again with the kit's iodinated mid-region human PTHrP antibody for 20 hours. Wells were washed and counted in a Packard Cobra II gamma counter. Ample values were extrapolated from the standard curve using GraphPad Prism software and a nonlinear regression curve fit to a sigmoidal dose 
response with a variable slope. Any given sample was defined as being above the limit of detection if it was both at least 2 SDs above the mean of 4 zero standards and if it fell within the linear portion of the standard curve. The analyses involved 5 to 9 mice per group.

Milk calcium concentration. Serum milk calcium concentration was determined using the Quantichrom Calcium Assay Kit (DICA500, BioAssay Systems) according to the manufacturer's protocol. In brief, milk was diluted 1:100 in distilled water before measuring calcium concentration, and the level of milk concentration was normalized to the protein content, which was measured using the Bradford protein determination assay (Bio-Rad Laboratories). The analyses involved 4-6 lactating Ctsk ${ }^{f / f l}$ and $C t s k^{o c y}$ mice.

Statistics. Multiple comparisons were analyzed using a 2-way ANOVA followed by Fisher's protected least significant difference (PLSD) post hoc test comparing all groups. A 2-way ANOVA was used to determine an interaction between Ctsk and lactation. An unpaired, 2 -tailed Student $t$ test was used for comparisons of 2 groups. GraphPad Prism 7 software was used for statistical analyses. A P value of less than 0.05 was considered statistically significant.

Study approval. All animal procedures were performed in accordance with a protocol approved by the IACUC of Harvard Medical School and followed the US Public Health Service Policy on Humane Care and Use of Laboratory Animals.

\section{Author contributions}

SL, RB, and FG designed the study. SL, YI, and FG performed experiments. RK generated the floxed mice. SL, KN, VTC, LN, $\mathrm{NI}, \mathrm{DB}, \mathrm{DH}$, and PD performed measurements. VP maintained the mice colonies and collected samples. SL, FG, and RB wrote the manuscript. SL, RB, FG, JW, and MLB edited the manuscript.

\section{Acknowledgments}

The authors would like to thank J.Q. Feng (Texas A\&M University Baylor College of Dentistry, Dallas, Texas, USA) for the Dmp1-Cre-transgenic mice. The Ocy454 cell line was provided by P. Pajevic Divieti (Massachusetts General Hospital), thanks to a grant from the National Institute of Arthritis and Musculoskeletal and Skin Diseases (NIAMS), NIH (P30 AR066261). Confocal analysis was performed at the Harvard NeuroDiscovery Center. This work was supported in part by an investigator-initiated grant from Merck \& Co. Inc.

Address correspondence to: Roland Baron, Department of Medicine, Harvard Medical School, and Division of Bone and Mineral Research Department of Oral Medicine, Infection and Immunity, Harvard School of Dental Medicine, Boston, MA 02115. Phone: 617.432.7320; Email: roland_baron@hms.harvard.edu.
1. Duong le T, Leung AT, Langdahl B. Cathepsin K Inhibition: A New Mechanism for the Treatment of Osteoporosis. Calcif Tissue Int . 2016;98(4):381-397.

2. Duong LT. Therapeutic inhibition of cathepsin K-reducing bone resorption while maintaining bone formation. Bonekey Rep. 2012;1:67.

3. Saftig P, et al. Impaired osteoclastic bone resorption leads to osteopetrosis in cathepsin-K-deficient mice. Proc Natl Acad Sci U S A 1998;95(23):13453-13458.

4. Kiviranta R, et al. Impaired bone resorption in cathepsin K-deficient mice is partially compensated for by enhanced osteoclastogenesis and increased expression of other proteases via an increased RANKL/OPG ratio. Bone. 2005;36(1):159-172.

5. Pennypacker B, et al. Bone density, strength, and formation in adult cathepsin $\mathrm{K}(-/-)$ mice. Bone. 2009;44(2):199-207.

6. Lotinun S, et al. Osteoclast-specific cathepsin K deletion stimulates S1P-dependent bone formation. J Clin Invest. 2013;123(2):666-681.

7. Everts V, et al. Cathepsin K deficiency in pycnodysostosis results in accumulation of non-digested phagocytosed collagen in fibroblasts. Calcif Tissue Int. 2003;73(4):380-386.

8. Hou WS, et al. Characterization of novel cathepsin K mutations in the pro and mature polypeptide regions causing pycnodysostosis. J Clin Invest. 1999;103(5):731-738.

9. Motyckova G, Fisher DE. Pycnodysostosis: role and regulation of cathepsin $\mathrm{K}$ in osteoclast function and human disease. Curr Mol Med. 2002;2(5):407-421.

10. Baud CA. [Morphology and inframicroscopic structure of osteocytes]. Acta Anat (Basel). 1962;51:209-225.

11. Belanger LF, Robichon J, Migicovsky BB, Copp
DH, Vincent J. Resorption without osteoclasts (osteolysis). In: Sognnaes RF, ed. Mechanism of hard tissue destruction. Washington, DC, USA: American Association for the Advancement of Science; 1963:531-556.

12. Bélanger LF. Osteocytic osteolysis. Calcif Tissue Res. 1969;4(1):1-12.

13. Lean JM, Mackay AG, Chow JW, Chambers TJ. Osteocytic expression of mRNA for c-fos and IGF-I: an immediate early gene response to an osteogenic stimulus. Am J Physiol.1996; 270(6 Pt 1):E937-E945.

14. Teti A, Zallone A. Do osteocytes contribute to bone mineral homeostasis? Osteocytic osteolysis revisited. Bone. 2009;44(1):11-16.

15. Wysolmerski JJ. Osteocytes remove and replace perilacunar mineral during reproductive cycles. Bone. 2013;54(2):230-236.

16. Bonewald LF. The role of the osteocyte in bone and nonbone disease. Endocrinol Metab Clin North Am. 2017;46(1):1-18.

17. Dole NS, et al. Osteocyte-intrinsic TGF-beta signaling regulates bone quality through perilacunar/canalicular remodeling. Cell Rep. 2017;21(9):2585-2596.

18. Qing H, et al. Demonstration of osteocytic perilacunar/canalicular remodeling in mice during lactation. J Bone Miner Res. 2012;27(5):1018-1029.

19. Jähn K, et al. Osteocytes acidify their microenvironment in response to $\mathrm{PTHrP}$ in vitro and in lactating mice in vivo.J Bone Miner Res. 2017;32(8):1761-1772.

20. Kovacs CS. The skeleton is a storehouse of mineral that is plundered during lactation and (fully?) replenished afterwards. J Bone Miner Res. 2017;32(4):676-680.

21. Ritchie LD, et al. A longitudinal study of calcium homeostasis during human pregnancy and lactation and after resumption of menses. Am J Clin
Nutr. 1998;67(4):693-701.

22. Specker BL, Tsang RC, Ho ML. Changes in calcium homeostasis over the first year postpartum: effect of lactation and weaning. Obstet Gynecol. 1991;78(1):56-62.

23. VanHouten JN, et al. Mammary-specific deletion of parathyroid hormone-related protein preserves bone mass during lactation. J Clin Invest. 2003;112(9):1429-1436.

24. Ardeshirpour L, Brian S, Dann P, VanHouten J, Wysolmerski J. Increased PTHrP and decreased estrogens alter bone turnover but do not reproduce the full effects of lactation on the skeleton. Endocrinology. 2010;151(12):5591-5601.

25. Ardeshirpour L, et al. Weaning triggers a decrease in receptor activator of nuclear factor-kappaB ligand expression, widespread osteoclast apoptosis, and rapid recovery of bone mass after lactation in mice. Endocrinology. 2007;148(8):3875-3886.

26. Bowman BM, Miller SC. Skeletal mass, chemistry, and growth during and after multiple reproductive cycles in the rat. Bone. 1999;25(5):553-559.

27. Kaya S, et al. Lactation-Induced Changes in the Volume of Osteocyte Lacunar-Canalicular Space Alter Mechanical Properties in Cortical Bone Tissue. J Bone Miner Res. 2017;32(4):688-697.

28. Bellido T, Saini V, Pajevic PD. Effects of PTH on osteocyte function. Bone. 2013;54(2):250-257.

29. Nakashima T, et al. Evidence for osteocyte regulation of bone homeostasis through RANKL expression. Nat Med. 2011;17(10):1231-1234.

30. Poole KE, et al. Sclerostin is a delayed secreted product of osteocytes that inhibits bone formation. FASEB J. 2005;19(13):1842-1844.

31. Tatsumi S, et al. Targeted ablation of osteocytes induces osteoporosis with defective mechanotransduction. Cell Metab. 2007;5(6):464-475.

32. Tazawa K, Hoshi K, Kawamoto S, Tanaka M, 
Ejiri S, Ozawa H. Osteocytic osteolysis observed in rats to which parathyroid hormone was continuously administered. J Bone Miner Metab. 2004;22(6):524-529.

33. Xiong J, Onal M, Jilka RL, Weinstein RS, Manolagas SC, O'Brien CA. Matrix-embedded cells control osteoclast formation. Nat Med. 2011;17(10):1235-1241.

34. Fowler TW, et al. Glucocorticoid suppression of osteocyte perilacunar remodeling is associated with subchondral bone degeneration in osteonecrosis. Sci Rep. 2017;7:44618.

35. Cui Y, et al. Lrp5 functions in bone to regulate bone mass. Nat Med.2011;17(6):684-691.

36. Tang SY, Herber RP, Ho SP, Alliston T. Matrix metalloproteinase-13 is required for osteocytic perilacunar remodeling and maintains bone fracture resistance. J Bone Miner Res. 2012;27(9):1936-1950.

37. Dallas SL, Prideaux M, Bonewald LF. The osteocyte: an endocrine cell... and more. Endocr Rev. 2013;34(5):658-690.

38. Ansari N, et al. Autocrine and paracrine regulation of the murine skeleton by osteocyte-derived parathyroid hormone-related protein. J Bone Miner Res. 2018;33(1):137-153.

39. Spatz JM, et al. The Wnt inhibitor sclerostin is up-regulated by mechanical unloading in osteocytes in vitro. J Biol Chem. 2015;290(27):16744-16758.

40. Wysolmerski JJ. Interactions between breast, bone, and brain regulate mineral and skeletal metabolism during lactation. Ann N Y Acad Sci. 2010;1192:161-169.

41. Halloran BP, DeLuca HF. Skeletal changes during pregnancy and lactation: the role of vitamin $\mathrm{D}$. Endocrinology. 1980;107(6):1923-1929.
42. Kovacs CS. Maternal mineral and bone metabolism during pregnancy, lactation, and post-weaning recovery. Physiol Rev. 2016;96(2):449-547.

43. Belanger LF, Robichon J, Migicovsky BB, Copp $\mathrm{DH}$, Vincent J. Resorption without osteoclasts (osteolysis). In: Sognnaes RF, ed. Mechanism of hard tissue destruction. Washington, DC, USA: AAAS; 1963:531-556.

44. Shimada T, et al. FGF-23 is a potent regulator of vitamin D metabolism and phosphate homeostasis. J Bone Miner Res. 2004;19(3):429-435.

45. Gillies BR, et al. Absence of calcitriol causes increased lactational bone loss and lower milk calcium but does not impair post-lactation bone recovery in Cyp27b1 null mice.J Bone Miner Res. 2018;33(1):16-26.

46. Ardeshirpour L, et al. OPG Treatment prevents bone loss during lactation but does not affect milk production or maternal calcium metabolism. Endocrinology. 2015;156(8):2762-2773.

47. Kalkwarf HJ, Specker BL, Heubi JE, Vieira NE, Yergey AL. Intestinal calcium absorption of women during lactation and after weaning. Am J Clin Nutr. 1996;63(4):526-531.

48. Rolvien T, et al. Vitamin D regulates osteocyte survival and perilacunar remodeling in human and murine bone. Bone. 2017;103:78-87.

49. Tang SY, Alliston T. Regulation of postnatal bone homeostasis by TGF $\beta$. Bonekey Rep. 2013;2:255.

50. Zhang D, Leung N, Weber E, Saftig P, Brömme D. The effect of cathepsin K deficiency on airway development and TGF- $\beta 1$ degradation. Respir Res. 2011;12:72.

51. Fuller K, et al. Cathepsin K inhibitors prevent matrix-derived growth factor degradation by human osteoclasts. Bone. 2008;42(1):200-211.

52. Asagiri M, et al. Cathepsin K-dependent toll-like receptor 9 signaling revealed in experimental arthritis. Science. 2008;319(5863):624-627.

53. Van Gool B, Dedieu S, Emonard H, Roebroek AJ. The matricellular receptor LRP1 forms an interface for signaling and endocytosis in modulation of the extracellular tumor environment. Front Pharmacol. 2015;6:271.

54. Yamamoto K, Murphy G, Troeberg L. Extracellular regulation of metalloproteinases. Matrix Biol. 2015;44-46:255-263.

55. Lu Y, Xie Y, Zhang S, Dusevich V, Bonewald LF, Feng JQ. DMP1-targeted Cre expression in odontoblasts and osteocytes. JDent Res. 2007;86(4):320-325.

56. Bouxsein ML, Boyd SK, Christiansen BA, Guldberg RE, Jepsen KJ, Müller R. Guidelines for assessment of bone microstructure in rodents using micro-computed tomography. J Bone Miner Res. 2010;25(7):1468-1486.

57. Dempster DW, et al. Standardized nomenclature, symbols, and units for bone histomorphometry: a 2012 update of the report of the ASBMR Histomorphometry Nomenclature Committee. J Bone Miner Res. 2013;28(1):2-17.

58. Turner CH, Burr DB. Basic biomechanical measurements of bone: a tutorial. Bone. 1993;14(4):595-608.

59. Livak KJ, Schmittgen TD. Analysis of relative gene expression data using real-time quantitative PCR and the 2(-Delta Delta C(T)) Method. Methods. 2001;25(4):402-408.

60. Jáuregui EJ, et al. Parallel mechanisms suppress cochlear bone remodeling to protect hearing. Bone. 2016;89:7-15.

61. Mamillapalli R, et al. Mammary-specific ablation of the calcium-sensing receptor during lactation alters maternal calcium metabolism, milk calcium transport, and neonatal calcium accrual. Endocrinology. 2013;154(9):3031-3042. 